\title{
Bounding Regions to Plane Steepest Descent Curves of Quasiconvex Families
}

\author{
Marco Longinetti, ${ }^{1}$ Paolo Manselli, ${ }^{1}$ and Adriana Venturi ${ }^{2}$ \\ ${ }^{1}$ Dipartimento di Matematica e Informatica Ulisse Dini, Università degli Studi di Firenze, Viale Morgagni 67/a, 50134 Firenze, Italy \\ ${ }^{2}$ Dipartimento GESAAF, Università degli Studi di Firenze, Piazzale delle Cascine 15, 50144 Firenze, Italy
}

Correspondence should be addressed to Marco Longinetti; marco.longinetti@unifi.it

Received 26 February 2016; Accepted 17 April 2016

Academic Editor: Wenyu Sun

Copyright (c) 2016 Marco Longinetti et al. This is an open access article distributed under the Creative Commons Attribution License, which permits unrestricted use, distribution, and reproduction in any medium, provided the original work is properly cited.

Two-dimensional steepest descent curves (SDC) for a quasiconvex family are considered; the problem of their extensions (with constraints) outside of a convex body $K$ is studied. It is shown that possible extensions are constrained to lie inside of suitable bounding regions depending on $K$. These regions are bounded by arcs of involutes of $\partial K$ and satisfy many inclusions properties. The involutes of the boundary of an arbitrary plane convex body are defined and written by their support function. Extensions SDC of minimal length are constructed. Self-contracting sets (with opposite orientation) are considered: necessary and/or sufficient conditions for them to be subsets of SDC are proved.

\section{Introduction}

Let $u$ be a smooth function defined in a convex body $\Omega \subset \mathbb{R}^{n}$. Let $D u(x) \neq 0$ in $\{x \in \Omega: u(x)>\min u\}$. A classical steepest descent curve of $u$ is a rectifiable curve $s \rightarrow x(s)$ solution to

$$
\frac{d x}{d s}=-\frac{D u}{|D u|}(x(s)) .
$$

Classical steepest descent curves are the integral curves of a unit field normal to the sublevel sets of the given smooth function $u$. We are interested in "generalized" steepest descent curves that are integral curves to a unit field normal to a nested family of convex sets $\left\{\Omega_{t}\right\}$ (see Definition 5); $\left\{\Omega_{t}\right\}$ will be called a quasiconvex family as in [1]. Sharp bounds about the length of the steepest descent curves for a quasi convex family have been proved in [2-4]. The geometry of these curves, equivalent definitions, related questions and generalizations have been studied in [5-9].

In the present work generalized steepest descent curves for a quasiconvex family (SDC for short) are defined as bounded oriented rectifiable curves $\gamma \subset \mathbb{R}^{n}$, with a locally Lipschitz continuous parameterization $T \ni t \rightarrow x(t)$, with ascent parameter, satisfying

$$
\langle\dot{x}(t), x(\tau)-x(t)\rangle \leq 0, \quad \text { a.e. } t \in T, \forall \tau \leq t ;
$$

$\langle\cdot, \cdot\rangle$ is the scalar product in $\mathbb{R}^{n}$. Let ordering $\preceq$ be chosen on $\gamma$, according to the orientation; let us denote

$$
\gamma_{x}=\{y \in \gamma: y \preceq x\} .
$$

In [8, Theorem 4.10], the SDC are characterized in an equivalent way as self-distancing curves, namely, oriented $(\preceq)$ continuous curves with the property that the distance of $x$ to an arbitrarily fixed previous point $x_{1}$ is not decreasing:

$$
\begin{aligned}
x_{1} & \leq x_{2} \preceq x_{3} \Longrightarrow \\
\left|x_{2}-x_{1}\right| & \leq\left|x_{3}-x_{1}\right| \quad \forall x_{1}, x_{2}, x_{3} \in \gamma .
\end{aligned}
$$

Thus steepest descent curves are self-distancing curves and both denoted SDC. In [8] self-distancing curves are called self-expanding curves. With the opposite orientation these curves have been also introduced, studied, and called selfapproaching curves (see [2]) or self-contracting curves (see [7]). 
In our work we are interested in the behaviour and properties of a plane SDC $\gamma$ beyond its final point $x_{0}$. One of the principal goals of the paper is to show that conditions (2) and (4) imply constraints for possible extensions of curve $\gamma$ beyond $x_{0}$; these constraints are written as bounding regions for the possible extensions of $\gamma_{x_{0}}$.

An important property that will be used later is the property of distancing from a set $A$.

Definition 1. Given a set $A$, an absolutely continuous curve $\gamma$, $T \ni t \rightarrow x(t)$ has the distancing from $A$ property if it satisfies

$$
\langle\dot{x}(t), y-x(t)\rangle \leq 0, \quad \text { a.e. } t \in T, \forall y \in A \text {. }
$$

Let us outline the content of our work. In Section 2 introductory definitions are given and covering maps for the boundary of a plane convex set, needed for later use, are introduced. In Section 3 the involutes of the boundary of a plane convex body are introduced and some of their properties are proved.

In Section 4 plane regions depending on the convex hull of $\gamma_{x_{0}}$ have been defined; these regions fence in or fence out the possible extensions of $\gamma_{x_{0}}$. The boundary of these sets consists of arcs of involutes of convex bodies, constructed in Section 3. As an application, in Section 4.1 the following problem has been studied: given a convex set $K, x_{0} \in \partial K, x_{1} \notin$ $K$, is it possible to construct SDC joining $x_{0}$ to $x_{1}$, satisfying the distancing from $K$ property? Minimal properties of this construction have been introduced and studied. In Section 5 sets of points more general than SDC are studied. A set $\sigma \subset$ $\mathbb{R}^{2}$ (not necessarily a curve) of ordered points satisfying (4) will be called self-distancing set; see also Definition 2; with the opposite order, $\sigma$ was called in [6] self-contracting set and many properties of these sets, as only subsets of selfcontracting curves, were there obtained. Another goal of the paper is the solution to the following question: given a selfdistancing set $\sigma \subset \mathbb{R}^{2}$ does a steepest descent curve $\gamma \supset$ $\sigma$ exist? In Section 5 examples, necessary and/or sufficient conditions are given when $\sigma$ consists of a finite or countable number of points $x_{i} \in \mathbb{R}^{2}$ and/or steepest descent curves $\gamma^{i} \subset \mathbb{R}^{2}$.

In the present work the two-dimensional case is studied. Similar results for the $n$-dimensional case are an open problem stated at the end of the work.

\section{Preliminaries and Definitions}

Let

$$
\begin{aligned}
B(z, \rho) & =\left\{x \in \mathbb{R}^{n}:|x-z|<\rho\right\}, \\
S^{n-1} & =\partial B(0,1)
\end{aligned}
$$

$$
n \geq 2
$$

A nonempty, compact convex set $K$ of $\mathbb{R}^{n}$ will be called a convex body. From now on, $K$ will always be a convex body not reduced to a point. Int $(K)$ and $\partial K$ denote the interior of $K$ and the boundary of $K,|\partial K|$ denotes its length, $\mathrm{cl}(K)$ is the closure of $K, \operatorname{Aff}(K)$ will be the smallest affine space containing $K$, and relint $K$ and $\partial_{\text {rel }} K$ are the corresponding subsets in the topology of $\operatorname{Aff}(K)$. For every set $S \subset \mathbb{R}^{n}, \operatorname{co}(S)$ is the convex hull of $S$. cone:

Let $q \in K$; the normal cone at $q$ to $K$ is the closed convex

$$
N_{K}(q)=\left\{x \in \mathbb{R}^{n}:\langle x, y-q\rangle \leq 0 \forall y \in K\right\} .
$$

When $q \in \operatorname{Int}(K)$, then $N_{K}(q)$ reduces to zero.

The tangent cone or support cone of $K$ at a point $q \in \partial K$ is given by

$$
T_{K}(q)=\mathrm{cl}\left(\bigcup_{y \in K}\{s(y-q): s \geq 0\}\right) .
$$

In two dimensions cones will be called sectors.

Let $K$ be a convex body and let $p$ be a point. A simple cap body $K^{p}$ is

$$
K^{p}=\bigcup_{0 \leq \lambda \leq 1}\{\lambda K+(1-\lambda) p\}=\operatorname{co}(K \cup\{p\}) .
$$

Cap bodies properties can be found in $[10,11]$.

2.1. Self-Distancing Sets and Steepest Descent Curves. Let us recall the following definitions.

Definition 2. Let us call self-distancing set a bounded subset $\sigma$ of $\mathbb{R}^{n}$, linearly ordered (by $\preceq$ ), with the property

$$
\begin{aligned}
x_{1} & \preceq x_{2} \preceq x_{3} \Longrightarrow \\
\left|x_{2}-x_{1}\right| & \leq\left|x_{3}-x_{1}\right|, \quad x_{1}, x_{2}, x_{3} \in \sigma .
\end{aligned}
$$

The self-distancing sets have been introduced in [6] with the opposite order. If a self-distancing set $\sigma$ is a closed connected set, not reduced to a point, then it can be proved that $\sigma$ is the support of a steepest descent curve $\gamma$ (see [8, Theorem 4.10, Theorem 4.8]) and it will also be called a selfdistancing curve $\gamma$.

The short name SDC will be used both for self-distancing curves and for steepest descent curves in all the paper.

Definition 3. Let $K$ be a convex body; $\gamma \subset \mathbb{R}^{2} \backslash$ relint $K$ will be called a self-distancing curve from $K$ (denoted $\mathrm{SDC}_{K}$ ) if

(i) $\gamma$ is a self-distancing curve,

(ii) $\gamma \cap \partial_{\text {rel }} K \neq \emptyset$,

(iii) $\gamma$ has the property

$$
\begin{aligned}
x & \leq x_{1} \Longrightarrow \\
|x-y| & \leq\left|x_{1}-y\right| \quad \forall y \in K, \forall x, x_{1} \in \gamma .
\end{aligned}
$$

When (ii) does not hold, that is $\gamma \cap \partial_{\text {rel }} K=\emptyset, \gamma$ will be called a deleted self-distancing curve from $K$.

Remark 4. Let $\gamma$ be $\mathrm{SDC}_{K}$, since $\gamma$ has an absolutely continuous parameterization ([8, Theorem 4.10 , Theorem 4.8$]$ ), thus property (11) for $\gamma$ is equivalent to (5). 
If $\gamma$ is SDC and $x \in \gamma$ then $\gamma \backslash \gamma_{x}$ is $\operatorname{SDC}_{\mathrm{co}\left(\gamma_{x}\right)}$. That is, the tangent vector $\dot{x}(t)$ to $\gamma$ is in the normal cone at $x(t)$ to the related convex set $\Omega_{t}:=\operatorname{co}\left(\gamma_{x(t)}\right)$. This condition is a generalization of the classicals steepest descent curves that are integral curves to a unit field normal to smooth quasiconvex families.

Nested families of convex sets have been introduced and studied by de Finetti [12] and Fenchel [1]. Let us recall some definitions.

Definition 5. Let $T$ be a real interval. A convex stratification (see [12]) is a nonempty family $\Omega$ of convex bodies $\Omega_{t} \subset \mathbb{R}^{n}$, $t \in T \subset \mathbb{R}$, linearly strictly ordered by inclusion $\left(\Omega_{1} \subset \Omega_{2}\right.$, $\left.\Omega_{1} \neq \Omega_{2}\right)$, with a maximum set $(\max \Re)$ and a minimum set $(\min \Omega)$.

Let $\mathfrak{\Omega}=\left\{\Omega_{t}\right\}_{t \in T}$ be a convex stratification. If for every $s \in T \backslash\{\max T\}$ the property

$$
\bigcap_{t>s} \Omega_{t}=\Omega_{s}
$$

holds, then as in [1], $\Re=\left\{\Omega_{t}\right\}_{t \in T}$ will be called a quasiconvex family.

An important quasiconvex family associated with a continuous self-distancing curve from $K, \gamma: t \rightarrow x(t)$ is $\mathbb{R}=$ $\left\{\Omega_{t}\right\}_{t \in T}$, where

$$
\Omega_{t}=\operatorname{co}\left(\gamma_{x(t)} \cup K\right) .
$$

The couple $(\gamma, \mathfrak{\Re})$ is special case of Expanding Couple, a class introduced in [8].

Remark 6. If $\gamma \in \mathrm{SDC}_{K}$, then for all $x \in \gamma$ the curve $\left(\gamma \backslash \gamma_{x}\right) \cup$ $\{x\}$ is a self-distancing curve from the convex hull of the set $\gamma_{x} \cup K$.

This fact is a direct consequence of the following.

Proposition 7 (see [8, Lemma 4.9]). Let $p, q, y_{i} \in \mathbb{R}^{n}, i=$ $1, \ldots$, s. If

$$
|p-y| \leq|q-y|, \quad \text { for } y=y_{i}, i=1, \ldots, s
$$

then the same holds for every $y \in \operatorname{co}\left(\left\{y_{i}, i=1, \ldots, s\right\}\right)$.

The statement of the previous proposition holds if large inequalities are replaced by strict inequalities everywhere.

\subsection{The Support Function of a Plane Convex Body. Let $K \subset$} $\mathbb{R}^{n}$ be a convex body not reduced to a point.

For a convex body $K$, the support function is defined as

$$
H_{K}(x)=\sup _{y \in K}\langle x, y\rangle, \quad x \in \mathbb{R}^{n},
$$

where $\langle\cdot, \cdot\rangle$ denotes the scalar product in $\mathbb{R}^{n}$. For $n=2, \vartheta \in \mathbb{R}$, let $\theta=(\cos \vartheta$, $\sin \vartheta) \in S^{1}$ and $h_{K}(\vartheta):=H_{K}(\theta)$; it will be denoted $h(\vartheta)$ if no ambiguity arises.

For every $\theta \in S^{1}$ there exists at least one point $x \in \partial K$ such that

$$
\langle\theta, y-x\rangle \leq 0 \quad \forall y \in K
$$

this means that the line through $x$ orthogonal to $\theta$ supports $K$. For every $x \in \partial K$ let $\widehat{N_{x}}$ be the set of $\theta \in S^{1}$ such that (16) holds. Let $F(\theta)$ be the set of all $x \in \partial K$ satisfying (16). If $\partial K$ is strictly convex at the direction $\theta$ then $F(\theta)$ reduces to one point and it will be denoted by $x(\theta)$.

Definition 8. The set valued map $G: \partial K \rightarrow S^{1}, \partial K \ni x \rightarrow$ $\widehat{N_{x}} \subset S^{1}$, is the generalized Gauss map; $x \in \partial K$ is a vertex on $\partial K$ iff $\widehat{N_{x}}$ is a sector with interior points. The set valued map $F: S^{1} \rightarrow \partial K, S^{1} \ni \theta \rightarrow F(\theta) \subset \partial K$ is the reverse generalized Gauss map; $F(\theta)$ is a closed segment, possibly reduced to a single point, and it will be called 1 -face when it has interior points.

Let $P$ be the covering map

$$
\begin{aligned}
P: & \mathbb{R} \longrightarrow S^{1}, \\
\mathbb{R} & \ni \vartheta \longrightarrow \\
\theta & =(\cos \vartheta, \sin \vartheta) \in S^{1} .
\end{aligned}
$$

Let $L=|\partial K|$, and let $s \rightarrow x_{l}(s), 0 \leq s<L\left(s \rightarrow x_{r}(s)\right.$, $0 \leq s<L$ ) be the parametric representations of $\partial K$ depending on the arc length counterclockwise (clockwise) with an initial point (not necessarily the same). Let us extend $x_{l}(\cdot)$ and $x_{r}(\cdot)$ by defining

$$
x_{l}(s):=x_{l}(s-k L) \quad \text { if } k L \leq s<(k+1) L,(k \in \mathbb{Z}),
$$

similarly for $x_{r}$.

Let us fix $x_{0} \in \partial K, \theta_{0} \in G\left(x_{0}\right), \theta_{0}=\left(\cos \vartheta_{0}\right.$, $\left.\sin \vartheta_{0}\right)$, and $\vartheta_{0} \in \mathbb{R}$.

For later use, we need to have $x_{0}=x_{l}\left(s_{0}\right)=x_{r}\left(s_{0}\right)$; this can be realized by choosing suitable initial points for the parameterizations $x_{l}$ and $x_{r}$.

Then

$$
x_{l}\left(s_{0}+s\right)=x_{r}\left(s_{0}+L-s\right), \quad \forall s \in \mathbb{R} .
$$

The maps

$$
\begin{aligned}
& x_{l}: \mathbb{R} \longrightarrow \partial K, \\
& x_{r}: \mathbb{R} \longrightarrow \partial K
\end{aligned}
$$

are covering maps.

The initial parameters will be

$$
\begin{aligned}
& x_{0}=x_{l}\left(s_{0}\right)=x_{r}\left(s_{0}\right) \in \partial K, \\
& S^{1} \ni \theta_{0} \in F^{-1}\left(x_{0}\right), \\
& \mathbb{R} \ni \vartheta_{0} \in P^{-1}\left(\theta_{0}\right)
\end{aligned}
$$

$\left(F^{-1}\left(x_{0}\right), P^{-1}\left(\theta_{0}\right)\right.$ are the back images of $F, P$, resp.). Let $k \in$ $\mathbb{Z}$. Let us define, for $\vartheta_{0}+2 k \pi<\vartheta<\vartheta_{0}+2(k+1) \pi$ :

$$
\begin{aligned}
& s_{l_{+}}(\vartheta):=\sup \left\{s \in \mathbb{R}: k L<s \leq(k+1) L, x_{l}(s)\right. \\
& \quad \in F(P(\vartheta))\} ;
\end{aligned}
$$


if $\vartheta=\vartheta_{0}+2 k \pi$

$$
\begin{aligned}
& s_{l+}(\vartheta):=\sup \left\{s \in \mathbb{R}: k L \leq s<(k+1) L, x_{l}(s)\right. \\
& \quad \in F(P(\vartheta))\} .
\end{aligned}
$$

Similarly, let us define for $\vartheta_{0}+2(k-1) \pi<\vartheta<\vartheta_{0}+2 k \pi$ :

$$
\begin{aligned}
& s_{r-}(\vartheta):=\inf \left\{s \in \mathbb{R}: k L \leq s<(k+1) L, x_{r}(s)\right. \\
& \quad \in F(P(\vartheta))\} ;
\end{aligned}
$$

if $\vartheta=\vartheta_{0}+2 k \pi$

$$
\begin{aligned}
& s_{r-}(\vartheta):=\inf \left\{s \in \mathbb{R}:(k-1) L<s \leq k L, x_{r}(s)\right. \\
& \quad \in F(P(\vartheta))\} .
\end{aligned}
$$

The function $s_{l_{+}}$is increasing in $\mathbb{R}$ and right continuous and with left limits (so called cadlag function). Similar properties hold for $-s_{r-}$. Let us recall that a cadlag increasing function $s(\vartheta), \vartheta \in \mathbb{R}$, has a right continuous inverse defined as

$$
\vartheta(s)=\inf \{\vartheta: s(\vartheta)>s\}
$$

Let $\vartheta_{l+}(\cdot)$ be the right continuous inverse of $s_{l+}(\cdot)$. Let $s \rightarrow$ $\vartheta_{r-}(s)$ be the opposite of the right continuous inverse of $-s_{r-}(\cdot)$.

Let us introduce for simplicity

$$
\begin{aligned}
\mathbf{n}_{\vartheta} & :=(\cos \vartheta, \sin \vartheta) \\
\mathbf{t}_{\vartheta} & :=(-\sin \vartheta, \cos \vartheta)
\end{aligned}
$$

Let $\vartheta \rightarrow h(\vartheta)$ be the support function of $K$.

It is well known ([13]) that if $\partial K$ is $C_{+}^{2}$ (i.e., $\partial K \in C^{2}$, with positive curvature), then $h$ is $C^{2}$ and the counterclockwise element $\operatorname{arc} d s$ of $\partial K$ is given by

$$
d s=(h+\ddot{h}) d \vartheta
$$

$h(\vartheta)+\ddot{h}(\vartheta)$ is the positive radius of curvature; moreover the reverse Gauss map $F: \theta \rightarrow x \in \partial K$ is a 1-1 map given by

$$
x(\theta):=h(\vartheta) \mathbf{n}_{\vartheta}+\dot{h}(\vartheta) \mathbf{t}_{\vartheta}, \quad \vartheta \in P^{-1}(\theta) .
$$

The previous formula also holds for an arbitrary convex body, for every $\vartheta$ such that $F(\theta)$ is reduced to a point; see [10]. Let us recall that a real valued function $x \rightarrow f(x)$ is called semiconvex on $\mathbb{R}$ when there exists a positive constant $C$ such that $f(x)+C x^{2}$ is convex on $\mathbb{R}$. From (29) the function $\vartheta \rightarrow$ $h(\vartheta)+(1 / 2) \vartheta^{2} \max h$ is convex on $\mathbb{R}$; thus $h$ is semiconvex. In the case that $K$ is an arbitrary convex body, by approximation arguments with $C_{+}^{2}$ convex bodies (see [11]) it follows that the support function of $K$ is also semiconvex. As consequence $h$ is Lipschitz continuous, it has left (right) derivative $\dot{h}_{-}$(resp., $\dot{h}_{+}$) at each point, which is left (right) continuous. Moreover at each point the right limit of $\dot{h}_{-}$is $\dot{h}_{+}$and the left limit of $\dot{h}_{+}$ is $\dot{h}_{-}$; see [14, pp. 228].
It is not difficult to show (from (30), with a right limit argument) that, for an arbitrary convex body, for $\vartheta \in \mathbb{R}$, the formula

$$
x_{l}\left(s_{l+}(\vartheta)\right)=h(\vartheta) \mathbf{n}_{\vartheta}+\dot{h}_{+}(\vartheta) \mathbf{t}_{\vartheta}
$$

holds. Similarly the formula

$$
x_{r}\left(s_{r-}(\vartheta)\right)=h(\vartheta) \mathbf{n}_{\vartheta}+\dot{h}_{-}(\vartheta) \mathbf{t}_{\vartheta}
$$

holds.

If $\partial K$ is not strictly convex at the direction $\theta=(\cos \vartheta$, $\sin \vartheta$ ) then $h$ is not differentiable at $\vartheta$ and

$$
\dot{h}_{+}(\vartheta)-\dot{h}_{-}(\vartheta)=\left|x_{l}\left(s_{l+}(\vartheta)\right)-x_{r}\left(s_{r-}(\vartheta)\right)\right|=|F(\theta)| \text {. }
$$

If $x_{1}, x_{2} \in \partial K$ let us define $\operatorname{arc}^{+}\left(x_{1}, x_{2}\right)$ as the set of points of $\partial K$ between $x_{1}$ and $x_{2}$ according to the counterclockwise orientation of $\partial K$ and $\operatorname{arc}^{-}\left(x_{1}, x_{2}\right)$ as the set of points between $x_{1}$ and $x_{2}$, according to the clockwise orientation; $\left|\operatorname{arc}^{ \pm}\left(x_{1}, x_{2}\right)\right|$ denote their length.

Remark 9. It is well known that a sequence of convex body $K^{(n)}$ converges uniformly to $K$ if and only if the corresponding sequence of support functions converges in the uniform norm; see [11, pp. 66]. Moreover as the two sequences of the end points of a closed counterclockwise oriented arc of $\partial K^{(n)}$ converge, then the sequence of the corresponding arcs converges to a connected arc of $\partial K$ and the sequence of the corresponding lengths converges too.

Proposition 10. Let $K$ be a convex body and $h$ its support function; then

$$
\begin{array}{r}
s_{l+}(\vartheta)-s_{l+}\left(\vartheta_{0}\right)=\int_{\vartheta_{0}}^{\vartheta} h(\tau) d \tau+\left(\dot{h}_{+}(\vartheta)-\dot{h}_{+}\left(\vartheta_{0}\right)\right), \\
\forall \vartheta \geq \vartheta_{0} ; \\
s_{r-}\left(\vartheta_{0}\right)-s_{r-}(\vartheta)=\int_{\vartheta_{0}}^{\vartheta} h(\tau) d \tau+\left(\dot{h}_{-}(\vartheta)-\dot{h}_{-}\left(\vartheta_{0}\right)\right), \\
\forall \vartheta \leq \vartheta_{0} .
\end{array}
$$

Proof. For every convex body $K$ not reduced to a point the function $\vartheta \rightarrow s_{l+}(\vartheta)$ is defined everywhere and satisfies the weak form of (29); namely,

$$
\begin{aligned}
-\int_{\mathbb{R}} s_{l+}(\eta) \dot{\phi}(\eta) d \eta=\int_{\mathbb{R}}(\phi+\ddot{\phi})(\eta) & h(\eta) d \eta, \\
& \forall \phi \in C_{0}^{\infty}(\mathbb{R}) .
\end{aligned}
$$

Using the fact that $\vartheta \rightarrow h(\vartheta)$ is Lipschitz continuous, integrating by parts (36), the formula

$$
\begin{aligned}
& -\int_{\mathbb{R}} s_{l+}(\eta) \dot{\phi}(\eta) d \eta \\
& \quad=-\int_{\mathbb{R}} \dot{\phi}(\eta)\left(\int_{0}^{\eta} h(\tau) d \tau+\dot{h}(\eta)\right) d \eta,
\end{aligned}
$$


holds. Thus

$$
s_{l+}(\eta)=c+\int_{0}^{\eta} h(\tau) d \tau+\dot{h}(\eta), \quad \text { a.e. }
$$

with $c$ constant. Passing to the right limit, the equality

$$
s_{l+}(\eta)=c+\int_{0}^{\eta} h(\tau) d \tau+\dot{h}_{+}(\eta), \quad \forall \eta \in \mathbb{R}
$$

holds. Formula (34) follows, by computing $s_{l+}(\vartheta)-s_{l+}\left(\vartheta_{0}\right)$, using the previous equality. Similarly (35) is proved.

\section{Involutes of a Closed Convex Curve}

Definition 11. Let $I$ be an interval. A plane curve $I \ni t \rightarrow x(t)$ is convex if at every point $x$ it has right tangent vector $T^{+}(x)$ and $\arg T^{+}(x(t))$ is not decreasing function.

Let $s \rightarrow x(s)$ be the arc length parameterization of a smooth curve; the classical definition of involute starting at a point $x_{0}=x\left(s_{0}\right)$ of the curve $x(\cdot)$ is

$$
i(s)=x(s)-\left(s-s_{0}\right) x^{\prime}(s) \quad s \geq s_{0} .
$$

Let us notice that $s$ is the arc length of the curve, not of the involute; if $s_{0}=0$, then the starting point of the involute coincides with the starting point of the curve. It is easy to construct an involute of a convex polygonal line (even if classical definition (40) does not work) by using arcs of circle centered at its corner points; moreover the involute depends on the orientation of the curve.

In this section, involutes for the boundary of an arbitrary plane convex body $K$, not reduced to a point, will be defined. The assumption that $K$ is an arbitrary convex body is needed to work with the involutes of the convex sets, not smooth, studied in Section 4.

Let $K \in C_{+}^{2}$; let $x_{0}$ be a fixed point of $\partial K ; s \rightarrow x(s)$ can be the clockwise parameterization of $\partial K$ or the counterclockwise parameterization. Since there exist two orientations, then two different involutes have to be considered. As noted previously one can assume that the parameterizations of $\partial K$ have been chosen so that $x_{0}=x_{l}\left(s_{0}\right)=x_{r}\left(s_{0}\right)$.

Definition 12. Let one denote by $i_{l, x_{0}}$ the left involute of $\partial K$ starting at $x_{0}$ corresponding to the counterclockwise parameterization of $\partial K$ and by $i_{r, x_{0}}$ the right involute corresponding to the clockwise parameterization. When one needs to emphasize the dependence on $K$ of involutes, they will be written as $i_{l, x_{0}}^{K}, i_{r, x_{0}}^{K}$.

Remark 13. Let us notice that if $\rho$ is a plane reflection with respect to a fixed axis then

$$
i_{r, x_{0}}^{K}=\rho\left(i_{l, \rho\left(x_{0}\right)}^{\rho(K)}\right)
$$

This relation allows us to prove our results for the left involutes only and to state without proof the analogous results for the right involutes.
Theorem 14. Let one fix the initial parameters $x_{0}, s_{0}, \theta_{0}$, and $\vartheta_{0}$. The left and the right involutes of a plane convex curve starting at $x_{0} \in \partial K$, boundary of a $C_{+}^{2}$ plane convex body $K$ with support function $h$, are parameterized by the value $\vartheta$ related to the outer normal $\mathbf{n}_{\vartheta}$ to $K$, as follows:

$$
\begin{array}{r}
i_{l, x_{0}}(\vartheta)=h(\vartheta) \mathbf{n}_{\vartheta}-\left(\int_{\vartheta_{0}}^{\vartheta} h(\tau) d \tau-\dot{h}\left(\vartheta_{0}\right)\right) \mathbf{t}_{\vartheta}, \\
\text { for } \vartheta \geq \vartheta_{0}, \\
i_{r, x_{0}}(\vartheta)=h(\vartheta) \mathbf{n}_{\vartheta}-\left(\int_{\vartheta_{0}}^{\vartheta} h(\tau) d \tau-\dot{h}\left(\vartheta_{0}\right)\right) \mathbf{t}_{\vartheta}, \\
\text { for } \vartheta \leq \vartheta_{0} .
\end{array}
$$

Proof. In the present case there is a 1-1 mapping between $\vartheta$ and $s$; from (29), it follows that

$$
s-s_{0}=\int_{\vartheta_{0}}^{\vartheta} h(\tau) d \tau+\dot{h}(\vartheta)-\dot{h}\left(\vartheta_{0}\right) ;
$$

then, changing the variable $s$ with $\vartheta$ in (40), with elementary computation, (42) is obtained (since $x^{\prime}(s)=\mathbf{t}_{9}$ and (30) holds). Formula (43) follows from (32) and (35).

For an arbitrary convex body $K$ in place of (30), formulas (31) and (32) have to be used.

Definition 15. Let $K$ be a plane convex body; let

$$
\begin{aligned}
& x_{0}=x\left(s_{0}\right) \in \partial K, \\
& \vartheta_{0}^{+}:=\vartheta_{l+}\left(s_{0}\right), \\
& s_{0}^{+}:=s_{l+}\left(\vartheta_{0}^{+}\right) .
\end{aligned}
$$

The left involute of $\partial K$ starting at $x_{0}$ will be defined as

$$
i_{l, x_{0}}(\vartheta)=x_{l}\left(s_{l+}(\vartheta)\right)-\left(s_{l+}(\vartheta)-s_{0}\right) \mathbf{t}_{\vartheta} \quad \text { for } \vartheta \geq \vartheta_{0}^{+} \text {; }
$$

similarly if $\vartheta_{0}^{-}:=\vartheta_{r-}\left(s_{0}\right), s_{0}^{-}:=s_{r-}\left(\vartheta_{0}^{-}\right)$, the right involute starting at $x_{0}$ will be defined as

$$
i_{r, x_{0}}(\vartheta)=x_{r}\left(s_{r-}(\vartheta)\right)+\left(s_{r-}(\vartheta)-s_{0}\right) \mathbf{t}_{\mathcal{\vartheta}} \quad \text { for } \vartheta \leq \vartheta_{0}^{-} .
$$

From (46) and (34) it follows that

$$
\begin{aligned}
i_{l, x_{0}}(\vartheta)= & h(\vartheta) \mathbf{n}_{\vartheta}-\left(\int_{\vartheta_{0}^{+}}^{\vartheta} h(\tau) d \tau-\dot{h}_{+}\left(\vartheta_{0}^{+}\right)\right) \mathbf{t}_{\vartheta} \\
& -\left|x_{0}-x_{l}\left(s_{0}^{+}\right)\right| \mathbf{t}_{\vartheta}, \quad \vartheta \geq \vartheta_{0}^{+} ;
\end{aligned}
$$

similarly from (47), (35) it follows that

$$
\begin{aligned}
i_{r, x_{0}}(\vartheta)= & h(\vartheta) \mathbf{n}_{\vartheta}-\left(\int_{\vartheta_{0}^{-}}^{\vartheta} h(\tau) d \tau-\dot{h}_{-}\left(\vartheta_{0}^{-}\right)\right) \mathbf{t}_{\vartheta} \\
& +\left|x_{0}-x_{r}\left(s_{0}^{-}\right)\right| \mathbf{t}_{\vartheta}, \quad \vartheta \leq \vartheta_{0}^{-} .
\end{aligned}
$$

Let us notice that in (48) and (49) the same parameter $\vartheta$ is used, but with different range; it turns out that $i_{l}$ is counterclockwise oriented; instead $i_{r}$ is clockwise oriented; $x_{0}=i_{l, x_{0}}\left(\vartheta_{0}^{+}\right)=i_{r, x_{0}}\left(\vartheta_{0}^{-}\right)$. 
Remark 16. The following facts can be derived from the above equations:

(i) since $h$ is Lipschitz continuous for every convex body $K$, then the involute $i_{l, x_{0}}$ is a rectifiable curve;

(ii) $i_{l, x_{0}}\left(\vartheta_{0}^{+}\right)=x_{0}$ and

$$
\left|i_{l, x_{0}}(\vartheta)-x_{l}\left(s_{l+}(\vartheta)\right)\right|=s_{l+}(\vartheta)-s_{0} ;
$$

(iii) if $x$ is a vertex of $\partial K$ then $i_{l, x_{0}}(\vartheta)$, for $(\cos \vartheta, \sin \vartheta) \epsilon$ $N_{K}(x)$, lies on an arc of circle centered at $x$ with radius $s_{l+}(\vartheta)-s_{0}$;

(iv) the involute (42) satisfies

$$
i_{l, x_{0}}(\vartheta+2 \pi)=i_{l, x_{0}}(\vartheta)-L \mathbf{t}_{\vartheta}, \quad \forall \vartheta \geq \vartheta_{0}^{+} .
$$

Lemma 17. Parameterization (48) of the involute $i_{l, x_{0}}$ is 1-1 in the interval $\left[\vartheta_{0}^{+}, \vartheta_{0}^{+}+2 \pi\right)$; moreover, except for at most a finite or countable set $\mathfrak{F}$ of values $\vartheta_{i}, i=1,2, \ldots$ (corresponding to the 1-face $F_{\theta_{i}}$ of $\left.\partial K\right), i_{l, x_{0}}$ is differentiable and

$$
\frac{d}{d \vartheta} i_{l, x_{0}}(\vartheta)=\left(s_{l+}(\vartheta)-s_{0}\right) \mathbf{n}_{\vartheta} \quad \text { for } \vartheta>\vartheta_{0}^{+}, \vartheta \notin \mathfrak{F}
$$

furthermore $i_{l, x_{0}}$ has left and right derivative with common direction $\mathbf{n}_{\vartheta}$ at $\vartheta=\vartheta_{i} \in \mathfrak{F}$.

Proof. By differentiating (48) and using (34), equality (52) is proved. Similar argument, at $\vartheta=\vartheta_{i} \in \mathfrak{F}$, proves that $\mathbf{n}_{\vartheta}$ is the common direction of the left and right derivatives.

Remark 18. Let $\vartheta \rightarrow i_{l, x_{1}}(\vartheta), \vartheta \rightarrow i_{l, x_{2}}(\vartheta), x_{i}=x\left(s_{i}\right), i=1,2$, be left involutes of $K$. Since

$$
\begin{aligned}
i_{l, x_{2}}(\vartheta)-i_{l, x_{1}}(\vartheta)=\left(s_{2}-s_{1}\right) \mathbf{t}_{\vartheta}, & \\
& \text { for } \vartheta>\max \left\{\vartheta_{l}^{+}\left(s_{2}\right), \vartheta_{l}^{+}\left(s_{1}\right)\right\},
\end{aligned}
$$

then they will be called parallel curves. Moreover, by (51), $i_{l, x_{0}}(\vartheta)$ and $i_{l, x_{0}}(\vartheta+2 \pi)$ will also be called parallel.

Theorem 19. If $d \varrho$ is the arc element of the involute $i_{l, x_{0}}$ then $\vartheta \rightarrow \varrho(\vartheta)$ is continuous and invertible in $\vartheta \geq \vartheta_{0}^{+}$with continuous inverse $[0,+\infty) \ni \varrho \rightarrow \vartheta(\varrho)$. Moreover

$$
d \varrho=\left(s_{l_{+}}(\vartheta)-s_{0}\right) d \vartheta \quad \text { for } \vartheta \geq \vartheta_{0}^{+}, \vartheta \notin \mathfrak{F}
$$

the involute is a convex curve with positive curvature a.e.:

$$
\frac{d \vartheta}{d \varrho}=\frac{1}{\left(s_{l+}(\vartheta)-s_{0}\right)} \quad \text { for } \vartheta>\vartheta_{0}^{+}, \vartheta \notin \mathfrak{\mho} \text {, }
$$

$\varrho \rightarrow i_{l, x_{0}}(\vartheta(\varrho))$ is $C^{1}$ everywhere, and

$$
\frac{d}{d \varrho} i_{l, x_{0}}=\mathbf{n}_{\vartheta(\varrho)}
$$

Moreover the following properties hold.

(i) For every $\varrho>0$ the right derivative

$$
\left(\frac{d \vartheta}{d \varrho}\right)^{+}=\frac{1}{s_{l+}(\vartheta(\varrho))-s_{0}}
$$

exists everywhere and it is a decreasing cadlag function.

(ii) $(d / d \varrho) i_{l, x_{0}}$ has everywhere right derivative given by

$$
\left(\frac{d^{2}}{d \varrho^{2}} i_{l, x_{0}}\right)^{+}=-\frac{1}{s_{l+}(\vartheta(\varrho))-s_{0}} \mathbf{t}_{\vartheta(\varrho)} .
$$

Theorem 20. Let $K^{(n)}$ be a sequence of plane convex bodies which converges uniformly to $K, x^{(n)} \in \partial K^{(n)}, x^{(n)} \rightarrow x_{0}$; then the corresponding sequences of left involutes $i_{l, x^{(n)}}^{K^{(n)}}$ converge uniformly to $i_{l, x_{0}}$ in compact subsets of $\left[\vartheta_{0}^{+},+\infty\right]$; moreover the corresponding sequence of their derivatives (with respect to the arc length) converges uniformly to $(d / d \varrho) i_{l, x_{0}}$.

Proof. By Remark 9 the sequence of functions $s_{l+}^{n}$ converges to $s_{l+}$. From (54) the arclengths of the left involutes $i_{l, x^{(n)}}^{K^{(n)}}$

$$
\varrho^{(n)}(\vartheta)=\int_{\vartheta_{0}}^{\vartheta}\left(s_{l+}^{(n)}(\vartheta)-s_{0}^{(n)}\right) d \vartheta
$$

converges uniformly in compact subsets of $\left[\vartheta_{0}^{+},+\infty\right)$ to the arc length $\varrho(\vartheta)$ of $i_{l, x_{0}}$; from (56) the same fact holds for their derivatives.

Let us consider the arc of the involute

$$
\eta:=\left\{i_{l, x_{0}}(\vartheta): \vartheta_{0}^{+} \leq \vartheta \leq \vartheta_{0}^{+}+3 \pi / 2\right\}
$$

and the set valued map $F$ (Definition 8). Let

$$
\begin{array}{r}
Q \\
=\bigcup_{\vartheta_{0}^{+} \leq \vartheta \leq \vartheta_{0}^{+}+3 \pi / 2}\left\{\lambda F(\theta)+(1-\lambda) i_{l, x_{0}}(\vartheta), 0 \leq \lambda \leq 1\right\}, \\
\theta=(\cos \vartheta, \sin \vartheta),
\end{array}
$$

the union of segments joining the points of $\eta$ with the corresponding points on $\partial K$.

Definition 21. If the tangent sector $T\left(x_{0}\right)$ to $K$ has an opening less than or equal to $\pi / 2$ as in Figure 1, then $Q \cup K$ is convex; let one define

$$
\vartheta_{l}^{*}=\vartheta_{0}^{+}+3 \pi / 2
$$

If $Q \cup K$ is not convex then let us consider $\operatorname{co}(Q \cup K)$. Let us notice that $\partial \operatorname{co}(Q \cup K) \backslash \partial(Q \cup K)$ is an open segment with end points $A, B$, with $A \in \eta, B \in \partial K$. Let us define $\vartheta_{l}^{*}$, with $\vartheta_{0}^{+}+3 \pi / 2 \leq \vartheta_{l}^{*}<\vartheta_{0}^{+}+2 \pi$ such that (see Figure 2) $\theta_{l}^{*}=$ $\left(\cos \vartheta_{l}^{*}, \sin \vartheta_{l}^{*}\right)$ is orthogonal to $A B, B \in F\left(\theta_{l}^{*}\right)$. Let $\vartheta_{1, l}$ be the smallest $\theta>\theta_{0}^{+}$satisfying $A=i_{l, x_{0}}\left(\vartheta_{1, l}\right)$. Clearly $\vartheta_{l}^{*}=$ $\vartheta_{1, l}+(3 / 2) \pi$. 


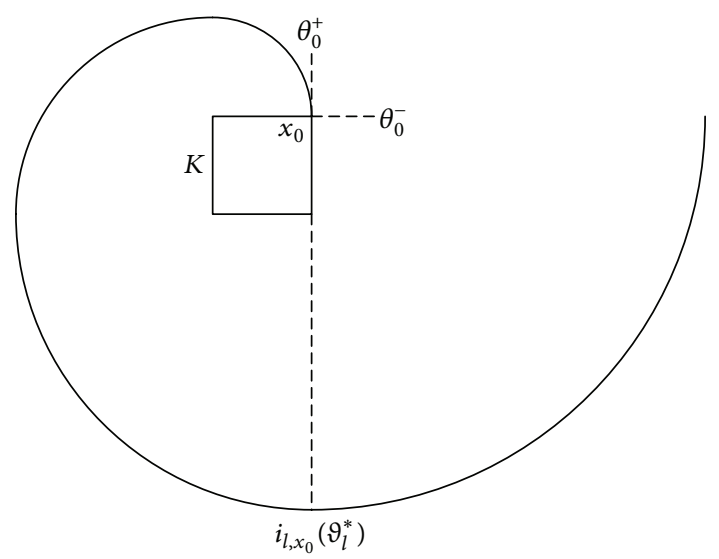

FIGURE 1: Left involute of a square.

For the right involutes a value $\vartheta_{r}^{*}$ is defined similarly, with $\vartheta_{0}^{-}-2 \pi<\vartheta_{r}^{*} \leq \vartheta_{0}^{-}-3 \pi / 2$, such that the line orthogonal to $\theta_{r}^{*}$ supporting $K$ at $F\left(\theta_{r}^{*}\right)$ is tangent to the right involute at $i_{r, x_{0}}\left(\vartheta_{1, r}\right)$ (see Figure 3 ) where $F\left(\theta_{r}^{*}\right)$ is the point $x\left(s_{r-}\left(\vartheta_{r}^{*}\right)\right)$, written as $x\left(\vartheta_{r}^{*}\right)$ for short.

Theorem 22. Let $i_{l}:=i_{l, x_{0}}$ be the left involute starting at $x_{0}$ on the boundary of a plane convex body $K$; then

(i) the left involute $\vartheta \rightarrow i_{l}(\vartheta)$ has the distancing from $K$ property for $\vartheta \geq \vartheta_{0}^{+}$but is not SDC for $\vartheta \geq \vartheta_{l}^{*}$;

(ii) the curve $\vartheta \in\left[\vartheta_{0}^{+}, \vartheta_{l}^{*}\right] \rightarrow i(\vartheta)$ is $S D C$;

(iii) for $y \in \operatorname{Int}(K)$ the distance function $J_{y}(\vartheta)=\left|i_{l}(\vartheta)-y\right|$ is strictly increasing for $\vartheta \geq \vartheta_{0}^{+}$;

(iv) if $y \in \partial K$, then $J_{y}(\vartheta)$ is not decreasing for $\vartheta \geq \vartheta_{0}^{+}$and $(d / d \vartheta) J>0$ for $(\cos \vartheta, \sin \vartheta) \notin N_{K}(y)$.

Proof. As $i_{l}$ is rectifiable, then the function $J_{y}^{2}(\vartheta)=\left|i_{l}(\vartheta)-y\right|^{2}$ is an absolutely continuous function for $\vartheta \geq \vartheta_{0}^{+}$, and from (52) for $\vartheta \notin \mathfrak{F}$

$$
\begin{aligned}
& \frac{1}{2} \frac{d}{d \vartheta} J_{y}^{2}=\left\langle\frac{d}{d \vartheta} i_{l}, i_{l}(\vartheta)-y\right\rangle \\
& =\left\langle\left(s_{l}(\vartheta)-s_{0}\right) \mathbf{n}_{\vartheta}, x_{l}\left(s_{l+}(\vartheta)+\left(s_{l+}(\vartheta)-s_{0}\right) \mathbf{t}_{\vartheta}-y\right\rangle\right. \\
& =\left(s_{l+}(\vartheta)-s_{0}\right)\left\langle\mathbf{n}_{\vartheta}, x_{l}\left(s_{l+}(\vartheta)\right)-y\right\rangle \geq 0
\end{aligned}
$$

the last inequality holds since $\mathbf{n}_{\vartheta}$ is the outer normal to $\partial K$ at $x_{l}\left(s_{l+}(\vartheta)\right)$. Moreover the previous inequality is strict for all $\vartheta$ if $y \in \operatorname{Int}(K)$, and it is also a strict inequality for $y \in \partial K$ and $y \notin F(\theta)$. This proves (iii) and (iv). Then (i) follows from (iii) and Definition 1 of distancing from $K$ property for a curve. To prove (ii) let us recall that SDC satisfies (2); then one has to prove that the angle at $i_{l}(\vartheta)$ between the vector $i_{l}(\vartheta)-i_{l}(\tau)$, $\vartheta_{0}^{+}<\tau<\vartheta \leq \vartheta_{l}^{*}$, and $\mathbf{n}_{\vartheta}$, the tangent vector at $i_{l}(\vartheta)$, is greater than or equal to $\pi / 2$; this is equivalent to show that the half line $r_{\vartheta}$ through $i_{l}(\vartheta)$ and $x\left(s_{l+}(\vartheta)\right)$ orthogonal to $\mathbf{n}_{\vartheta}$ supports at $i_{l}(\vartheta)$ the arc of $i_{l}$ from $x_{0}$ to $i_{l}(\vartheta)$. By Definition 21 this is the case for all $\vartheta$ between $\vartheta_{0}^{+}$and $\vartheta_{l}^{*}$.

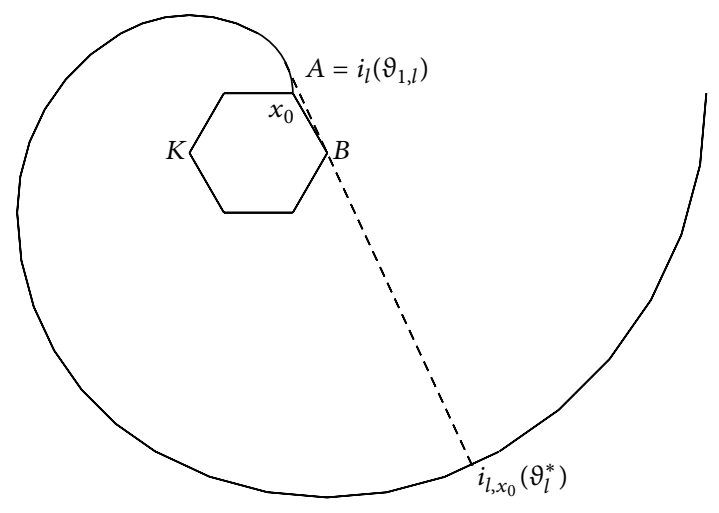

Figure 2: Left involute of an hexagon.

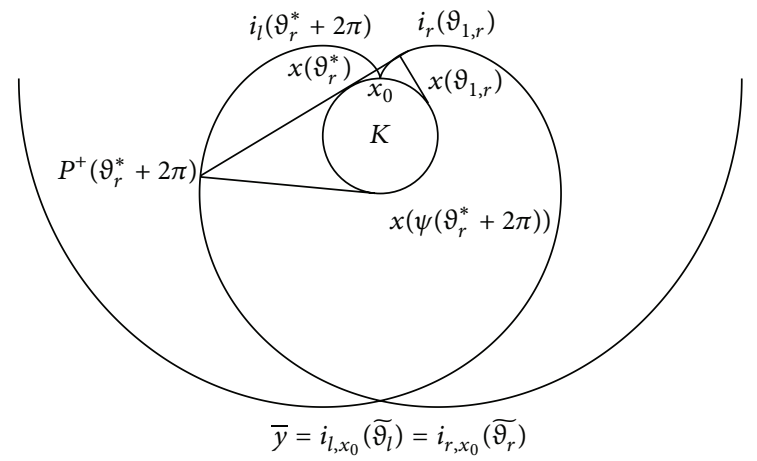

Figure 3: Involutes of a circumference.

Corollary 23. The left involute $\vartheta \rightarrow i_{l, x_{0}}(\vartheta)$ of the boundary of a plane convex body $K$ is a self-distancing curve from $K$ for $\vartheta \in\left[\vartheta_{0}^{+}, \vartheta_{l}^{*}\right]$; similarly right involute (49) is a self-contracting curve from $K$ for $\vartheta \in\left[\vartheta_{r}^{*}, \vartheta_{0}^{-}\right]$.

Proof. From (i) of Theorem 22 the left involute is a curve such that the distance of its points from all $y \in K$ is not decreasing; (ii) of the same theorem proves that it is a SDC. Let us recall that a self-contracting curve is a self-distancing curve with opposite orientation.

Theorem 24. Let $K$ be a plane convex body not reduced to a single point and let $x_{0}, s_{0}, \theta_{0}, \vartheta_{0}$ be the initial parameters. Let $\left[\vartheta_{0}^{+}, \vartheta_{0}^{+}+2 \pi\right] \ni \vartheta \rightarrow i_{l}(\vartheta)$ be an arc of the left involute starting at $x_{0}$, and let $\left[\vartheta_{0}^{-}-2 \pi, \vartheta_{0}^{-}\right] \ni \vartheta \rightarrow i_{r}(\vartheta)$ be an arc of the right involute ending at $x_{0}$; then there exists only one point $\bar{y} \neq x_{0}$ which belongs to both arcs and

$$
\bar{y}=i_{l}\left(\widetilde{\vartheta_{l}}\right)=i_{r}\left(\widetilde{\vartheta_{r}}\right)
$$

with

$$
\begin{aligned}
& \vartheta_{0}^{-} \leq \vartheta_{r}^{*}+2 \pi<\widetilde{\vartheta_{l}}<\vartheta_{0}^{+}+\frac{3 \pi}{2} \leq \vartheta_{l}^{*}, \\
& \vartheta_{r}^{*} \leq \vartheta_{0}^{-}-\frac{3 \pi}{2}<\widetilde{\vartheta_{r}}<\vartheta_{l}^{*}-2 \pi \leq \vartheta_{0}^{+} .
\end{aligned}
$$

Proof. For simplicity, first let us prove the existence of $\bar{y}$ assuming that $K \in C_{+}^{2}$. With the assumed conditions, 
$\mathbb{R} \ni \vartheta \rightarrow x(\vartheta):=x(\theta)$ defined by (30) is a parameterization of $\partial K$.

Let $\vartheta \in\left[\vartheta_{0}, \vartheta_{0}+2 \pi\right]$ and let $P^{+}(\vartheta)$ be the first common point of the half line $\left\{x(\vartheta)+\lambda \mathbf{t}_{9}, \lambda>0\right\}$ and of $i_{l}$. Moreover, let $\left[\vartheta_{0}, \vartheta_{l}^{*}\right] \ni \vartheta \rightarrow \psi(\vartheta)$ be the function satisfying

$$
P^{+}(\vartheta)=i_{l}(\psi(\vartheta)) .
$$

Let

$$
\phi(\vartheta):=\left|P^{+}(\vartheta)-i_{l}(\vartheta)\right|
$$

First the following sentence will be proved.

Claim 1. $P^{+}\left(\overline{\vartheta_{l}}\right)$ belongs to $i_{r}$ iff the equality

$$
\phi\left(\overline{\vartheta_{l}}\right)=L
$$

holds for some $\overline{\vartheta_{l}} \in\left[\vartheta_{0}, \vartheta_{0}+2 \pi\right], L=|\partial K|$.

Proof of Claim 1. If (68) holds, then

$$
\begin{aligned}
\left|P^{+}\left(\overline{\vartheta_{l}}\right)-x\left(\overline{\vartheta_{l}}\right)\right|= & \left|P^{+}\left(\overline{\vartheta_{l}}\right)-i_{l}\left(\overline{\vartheta_{l}}\right)\right| \\
& -\left|i_{l}\left(\overline{\vartheta_{l}}\right)-x\left(\overline{\vartheta_{l}}\right)\right| \\
= & L-\left|\operatorname{arc}^{+}\left(x_{0}, x\left(\overline{\vartheta_{l}}\right)\right)\right| \\
= & \left|\operatorname{arc}^{+}\left(x\left(\overline{\vartheta_{l}}\right), x_{0}\right)\right| \\
= & \left|\operatorname{arc}^{-}\left(x_{0}, x\left(\overline{\vartheta_{l}}-2 \pi\right)\right)\right| .
\end{aligned}
$$

Thus

$$
\begin{aligned}
P^{+}\left(\overline{\vartheta_{l}}\right) & =x\left(\overline{\vartheta_{l}}\right)+\left|\operatorname{arc}^{+}\left(x\left(\overline{\vartheta_{l}}\right), x_{0}\right)\right| \mathbf{t}_{\overline{\vartheta_{l}}} \\
& =x\left(\overline{\vartheta_{l}}-2 \pi\right)+\left|\operatorname{arc}^{-}\left(x_{0}, x\left(\overline{\vartheta_{l}}-2 \pi\right)\right)\right| \mathbf{t}_{\overline{\vartheta_{l}}-2 \pi} \\
& =i_{r}\left(\overline{\vartheta_{l}}-2 \pi\right) .
\end{aligned}
$$

Thus $P^{+}\left(\overline{\vartheta_{l}}\right)$ is on both arcs of involutes and the other way around.

Our aim is to prove that there exists $\overline{\vartheta_{l}} \in\left[\vartheta_{0}, \vartheta_{0}+3 \pi / 2\right]$ such that (68) holds. For this goal we prove next Claims 2 and 3.

Claim 2. The following facts hold in $\left[\vartheta_{0}, \vartheta_{l}^{*}\right]$ :

(i) $\psi$ is continuously differentiable and $\psi^{\prime}>0$;

(ii) $\phi^{\prime}>0$.

Proof of Claim 2. Let us prove that $\mathbf{n}_{\vartheta}$ and $\mathbf{n}_{\psi(\vartheta)}$ satisfy

$$
\left\langle\mathbf{n}_{\vartheta}, \mathbf{n}_{\psi(\vartheta)}\right\rangle<0
$$

Let us consider the triangle with vertices $x(\vartheta), i_{l}(\psi(\vartheta))$, $x(\psi(\vartheta))$. As

$$
\begin{aligned}
& \left|i_{l}(\psi(\vartheta))-x(\psi(\vartheta))\right|=\left|\operatorname{arc}^{+}\left(x_{0}, x(\psi(\vartheta))\right)\right| \\
& \quad \geq\left|\operatorname{arc}^{+}(x(\vartheta), x(\psi(\vartheta)))\right| \geq|x(\psi(\vartheta))-x(\vartheta)|,
\end{aligned}
$$

the angle between $x(\psi(\vartheta))-P^{+}(\vartheta)$ and $x(\vartheta)-P^{+}(\vartheta)$ is acute and the angle between $\mathbf{n}_{\vartheta}$ and $\mathbf{n}_{\psi(9)}$ is obtuse. Thus (71) follows. By definition, $\psi(\vartheta)$ solves (66); thus $\psi(\vartheta)$ is the implicit solution to

$$
\left\langle i_{l}(\psi(\vartheta))-x(\vartheta), \mathbf{n}_{\vartheta}\right\rangle=0 .
$$

As

$$
\left\langle\frac{d}{d \psi} i_{l}(\psi), \mathbf{n}_{\vartheta}\right\rangle=\left(s(\psi)-s_{0}\right)\left\langle\mathbf{n}_{\psi}, \mathbf{n}_{\vartheta}\right\rangle
$$

is negative by (71), then by Dini's Theorem equation (73) has a solution $\psi(\theta)$ satisfying

$$
\begin{aligned}
& \left(s(\psi)-s_{0}\right)\left\langle\mathbf{n}_{\psi}, \mathbf{n}_{\vartheta}\right\rangle \psi^{\prime}(\vartheta)+\left\langle i_{l}(\psi(\vartheta))-x(\vartheta), \mathbf{t}_{\vartheta}\right\rangle \\
& \quad=0 .
\end{aligned}
$$

As $i_{l}(\psi(\vartheta))-x(\vartheta)=\lambda \mathbf{t}_{\vartheta}(\lambda>0)$ and (71) holds, then $\psi^{\prime}>0$, and $\psi$ is strictly increasing and continuously differentiable.

Let us prove (ii).

The formula

$$
\begin{aligned}
& \frac{d}{d \vartheta}\left|i_{l}(\vartheta)-i_{l}(\psi(\vartheta))\right|^{2} \\
& \quad=2\left\langle i_{l}(\vartheta)-i_{l}(\psi(\vartheta)), \frac{d}{d \vartheta} i_{l}(\vartheta)-\frac{d}{d \vartheta} i_{l}(\psi(\vartheta))\right\rangle
\end{aligned}
$$

holds. Let us notice that $i_{l}(\vartheta)-i_{l}(\psi(\vartheta))$ is parallel to $\mathbf{t}_{9}$; thus by (52)

$$
\left\langle i_{l}(\vartheta)-i_{l}(\psi(\vartheta)), \frac{d}{d \vartheta} i_{l}(\vartheta)\right\rangle=0 .
$$

On the other hand

$$
\begin{aligned}
& -\left\langle i_{l}(\vartheta)-i_{l}(\psi(\vartheta)), \frac{d}{d \vartheta} i_{l}(\psi(\vartheta))\right\rangle \\
& =-\left\langle-s(\vartheta) \mathbf{t}_{\vartheta}-\lambda \mathbf{t}_{\vartheta},\left(s(\psi(\vartheta))-s_{0}\right) \mathbf{n}_{\psi(\vartheta)}\right\rangle \psi^{\prime} \\
& =(s(\vartheta)+\lambda)\left(s(\psi(\vartheta))-s_{0}\right)\left\langle\mathbf{t}_{\vartheta}, \mathbf{n}_{\psi(\vartheta)}\right\rangle \psi^{\prime} .
\end{aligned}
$$

As the angle between $\mathbf{t}_{\vartheta}$ and $\mathbf{n}_{\psi(\vartheta)}$ is acute, then last term in the above equalities is positive; thus the derivative in the left hand side of (76) is positive and (ii) of Claim 2 follows.

Claim 3. In the interval $\left[\vartheta_{0}, \vartheta_{l}^{*}\right]$ the function $\phi$ has values smaller than $L$ and greater than $L$.

Proof of Claim 3. The angles $\vartheta_{r}^{*}$ and $\vartheta_{1, r}$ have been introduced in Definition 21. For simplicity $x\left(s_{r-}\left(\vartheta_{1, r}\right)\right)$ will be denoted with $x\left(\vartheta_{1, r}\right)$. Let us consider the convex set bounded by $\operatorname{arc}^{+}\left(x\left(\psi\left(\vartheta_{r}^{*}+2 \pi\right)\right), x\left(\vartheta_{1, r}\right)\right)$ and by the polygonal line with vertices $x\left(\vartheta_{1, r}\right), i_{r}\left(\vartheta_{1, r}\right), P^{+}\left(\vartheta_{r}^{*}+2 \pi\right), x\left(\psi\left(\vartheta_{r}^{*}+2 \pi\right)\right)$; see Figure 3. 
Clearly the inequalities

$$
\begin{aligned}
&\left|i_{r}\left(\vartheta_{1, r}\right)-P^{+}\left(\vartheta_{r}^{*}+2 \pi\right)\right| \\
&<\mid\left|i_{r}\left(\vartheta_{1 r}\right)-x\left(\vartheta_{1, r}\right)\right| \\
&+\left|\operatorname{arc}^{-}\left(x\left(\vartheta_{1, r}\right), x\left(\psi\left(\vartheta_{r}^{*}+2 \pi\right)\right)\right)\right| \\
&+\left|x\left(\psi\left(\vartheta_{r}^{*}+2 \pi\right)\right)-P^{+}\left(\vartheta_{r}^{*}+2 \pi\right)\right| \\
&=\left|\operatorname{arc}^{-}\left(x_{0}, x\left(\vartheta_{1, r}\right)\right)\right| \\
&+\left|\operatorname{arc}^{-}\left(x\left(\vartheta_{1, r}\right), x\left(\psi\left(\vartheta_{r}^{*}+2 \pi\right)\right)\right)\right| \\
&+\left|\operatorname{arc}^{-}\left(x\left(\psi\left(\vartheta_{r}^{*}+2 \pi\right)\right), x_{0}\right)\right|=L
\end{aligned}
$$

hold. As

$$
\begin{aligned}
\phi\left(\vartheta_{r}^{*}+2 \pi\right) & =\left|i_{l}\left(\vartheta_{r}^{*}+2 \pi\right)-P^{+}\left(\vartheta_{r}^{*}+2 \pi\right)\right| \\
& <\left|i_{r}\left(\vartheta_{1, r}\right)-P^{+}\left(\vartheta_{r}^{*}+2 \pi\right)\right|,
\end{aligned}
$$

using the previous inequalities, one obtains

$$
\phi\left(\vartheta_{r}^{*}+2 \pi\right)<L
$$

Let us show now that

$$
\phi\left(\vartheta_{0}+\frac{3 \pi}{2}\right)>L
$$

holds.

Let $\rho$ be the half line with origin $x_{0}$ and direction $-\mathbf{t}_{\vartheta_{0}}$; $\rho-\left\{x_{0}\right\}$ crosses the arc $i_{r}$ in a first point $y_{1}=i_{r}\left(\alpha_{1}\right)$, with $\alpha_{1}<\vartheta_{0}-\pi / 2$. Then

$$
\begin{aligned}
r & :=\left|x_{0}-y_{1}\right|<\left|y_{1}-x\left(\alpha_{1}\right)\right|+\left|\operatorname{arc}^{-}\left(x\left(\alpha_{1}\right), x_{0}\right)\right| \\
& =L .
\end{aligned}
$$

The half line $\rho$ meets the arc $i_{1}$ in a point $y_{2}$ and $\left|y_{2}-x_{0}\right|=L$.

Property (iii) of Theorem 22 implies that the arc $D$ of the left involute after $y_{2}$ lies outside of the circle centered in $x_{0}$ and with radius $L$. Similar property for the right involute implies that the $\operatorname{arc} C$ of the right involute joining $x_{0}$ to $y_{1}$ lies in the circle with center $x_{0}$ and radius $r$; thus the straight line tangent to $K$ at $x\left(\vartheta_{0}+3 \pi / 2\right)$ meets the arc $C$ in $i_{r}\left(\vartheta_{0}-\pi / 2\right)$ and $D$ in $P^{+}\left(\vartheta_{0}+3 \pi / 2\right)$. Therefore

$$
\begin{aligned}
\phi\left(\vartheta_{0}+\frac{3}{2 \pi}\right)= & \left|i_{l}\left(\vartheta_{0}+\frac{3 \pi}{2}\right)-P^{+}\left(\vartheta_{0}+\frac{3 \pi}{2}\right)\right| \\
= & \left|i_{l}\left(\vartheta_{0}+\frac{3 \pi}{2}\right)-x\left(\vartheta_{0}+\frac{3 \pi}{2}\right)\right| \\
& +\left|x\left(\vartheta_{0}+\frac{3 \pi}{2}\right)-P^{+}\left(\vartheta_{0}+\frac{3 \pi}{2}\right)\right| \\
> & \left|i_{l}\left(\vartheta_{0}+\frac{3 \pi}{2}\right)-x\left(\vartheta_{0}+\frac{3 \pi}{2}\right)\right| \\
& +\left|x\left(\vartheta_{0}+\frac{3 \pi}{2}\right)-i_{r}\left(\vartheta_{0}-\frac{\pi}{2}\right)\right| \\
= & \left|\operatorname{arc}^{+}\left(x_{0}, x\left(\vartheta_{0}+\frac{3 \pi}{2}\right)\right)\right| \\
& +\left|\operatorname{arc}^{-}\left(x_{0}, x\left(\vartheta_{0}+\frac{3 \pi}{2}\right)\right)\right|=L .
\end{aligned}
$$

Inequality (82) is proved.
The intermediate values theorem implies that there exists $\overline{\vartheta_{l}} \in\left[2 \pi+\vartheta_{r}^{*}, \vartheta_{0}+3 \pi / 2\right]$ such that (68) holds. Claim 1 implies that

$$
P^{+}\left(\overline{\vartheta_{l}}\right)=i_{l}\left(\psi\left(\overline{\vartheta_{l}}\right)\right)=i_{r}\left(\overline{\vartheta_{l}}-2 \pi\right)
$$

so the right involute and the left involute meet each other in one point and (64) is proved with $\widetilde{\vartheta_{l}}=\psi\left(\overline{\vartheta_{l}}\right), \widetilde{\vartheta_{r}}=\overline{\vartheta_{l}}-2 \pi$.

By approximation argument the same result holds for an arbitrary convex body $K$.

Let us prove now that the point $\bar{y}$ is unique. Let us argue by contradiction. Let $P, Q$ be two distinct points on $i_{l} \cap i_{r}$, with $P \prec Q$ on $i_{l}$ and $i_{r}$; then since $i_{l}$ is a distancing curve from $x_{0}$,

$$
\left|P-x_{0}\right| \leq\left|Q-x_{0}\right|
$$

and since $i_{r}$ is a contracting curve to $x_{0}$,

$$
\left|P-x_{0}\right| \geq\left|Q-x_{0}\right|
$$

therefore all the points on the arc of $i_{l}$ and of $i_{r}$ between $P$ and $Q$ have the same distance from $x_{0}$; thus, between $P$ and $Q, i_{l}$ and $i_{r}$ (arc of involutes of a same convex body $K$ ) coincide with the same arc of circle centered at $x_{0}$; this implies that $K$ reduce to the point $x_{0}$, which is not possible for the assumption.

Definition 25. Let $z \notin K$. Let $z_{l}\left(z_{r}\right) \in \partial K$ on the contact set on the "left" (right) support line to $K$ through $z$. If the contact set is a 1-face on these support lines, then $z_{l}$ and $z_{r}$ are identified as the closest ones to $z$. The triangle $z z_{l} z_{r}$ is counterclockwise oriented.

Theorem 26. For every $\xi \in \partial K$ let one consider the left involutes $i_{l, \xi}$ and the right involutes $i_{r, \xi}$ parameterized by their arc length $\varrho$. The maps

$$
\begin{gathered}
\partial K \times(0,+\infty) \ni(\xi, \varrho) \longrightarrow \\
i_{l, \xi}(\theta(\varrho)) \in \mathbb{R}^{2} \backslash K, \\
\partial K \times(0,+\infty) \ni(\xi, \varrho) \longrightarrow \\
i_{r, \xi}(\theta(\varrho)) \in \mathbb{R}^{2} \backslash K
\end{gathered}
$$

are 1-1 maps.

Proof. Assume, in the proof, that $x_{0} \in \partial K, \theta_{0} \in G\left(x_{0}\right), \vartheta_{0}, s_{0}$ are fixed. Let $z \notin K$. The tangent sector to the cap body $K^{z}$ with vertex $z$ has two maximal segments $z z_{l}$ and $z z_{r}$ on the sides that do not meet $K$ (except at the end points $z_{l}$ and $z_{r}$ ). Let $\vartheta_{l}$ be such that $z_{l}=x_{l}\left(s_{l+}\left(\vartheta_{l}\right)\right)$, and let $\bar{s}$ be such that

$$
\left|z-z_{l}\right|=s_{l+}\left(\vartheta_{l}\right)-\bar{s} \text {. }
$$

Let $\xi_{l}=x_{l}(\bar{s})$; let $\bar{\vartheta}=\vartheta_{l}^{+}(\bar{s})$. From (50) and from the definition of left involute (46) (with $\xi_{l}$ in place of $x_{0}, \bar{\vartheta}$ in place of $\vartheta_{0}^{+}$, and $\bar{s}$ in place $\left.s_{0}\right)$ 
holds; thus the map $(\xi, \varrho) \rightarrow i_{l, \xi}(\varrho)$ is surjective. Moreover the map is also injective, since the left involutes do not cross each other since they are parallel (see Remark 18). Similar proof holds for the right involutes.

Let $\xi_{l}=x_{l}(\bar{s})$ be the starting point of the left involute $i_{l, \xi_{l}}$ through $z$, defined in the previous theorem; similarly let $\xi_{r}$ be the starting point of the right involute $i_{r, \xi}$ through $z$. Let us notice that $i_{l, \xi_{l}}$ and $i_{r, \xi_{r}}$ meet each other in a countable ordered set of points.

\section{1. $\mathfrak{J}$-Fence and $\mathfrak{G}$-Fence}

Definition 27. Let $K$ be a convex body in $\mathbb{R}^{2},|\partial K|>0, x_{0} \in$ $\partial K, \theta_{0} \in G\left(x_{0}\right), \theta_{0}=\left(\cos \vartheta_{0}, \sin \vartheta_{0}\right), s_{0} \in \mathbb{R}$. Let $i_{l}:=i_{l, x_{0}}$, and let $i_{r}:=i_{r, x_{0}}$. Let

$$
\bar{y}=i_{l}\left(\widetilde{\vartheta}_{l}\right)=i_{r}\left(\widetilde{\vartheta_{r}}\right) \in \mathbb{R}^{2} \backslash K
$$

be the first point where the two involutes cross each other (see Theorem 24). Let one define

$$
\begin{aligned}
& \mathfrak{\Im}_{l}\left(K, x_{0}\right):=\left\{y \in \mathbb{R}^{2}: y=t x_{0}+(1-t) i_{l}(\vartheta), 0 \leq t\right. \\
& \left.\quad \leq 1, \vartheta_{0}^{+} \leq \vartheta \leq \widetilde{\vartheta}_{l}\right\}, \\
& \mathfrak{\Im}_{r}\left(K, x_{0}\right):=\left\{y \in \mathbb{R}^{2}: y=t x_{0}+(1-t) i_{r}(\vartheta), 0 \leq t\right. \\
& \left.\quad \leq 1, \widetilde{\vartheta}_{r} \leq \vartheta \leq \vartheta_{0}^{-}\right\}, \\
& \mathfrak{\Im}\left(K, x_{0}\right):=\left(\mathfrak{\Im}_{l}\left(K, x_{0}\right) \cup \mathfrak{\Im}_{r}\left(K, x_{0}\right)\right) \backslash \operatorname{Int}(K) .
\end{aligned}
$$

$\mathfrak{\Im}\left(K, x_{0}\right)$ will be called the $\mathfrak{\Im}$-fence of $K$ at $x_{0}$.

Let us notice that $\mathfrak{\Im}_{l}\left(K, x_{0}\right)$ and $\mathfrak{\Im}_{r}\left(K, x_{0}\right)$ are two convex bodies in common with the segment $x_{0} \bar{y}$ only.

From Theorem 26 the starting point $\xi_{l}\left(\xi_{r}\right)$ of a left (right) involute is uniquely determined from any point $z \notin K$ of the involute. The arc of the points on the left (right) involute between the starting point and $z$ will be denoted by $i_{l, \xi_{l}}^{z}\left(i_{r, \xi_{r}}^{z}\right)$ or $i_{l}^{z}\left(i_{r}^{z}\right)$ for short. For $y \preceq w$ let us denote with $i_{l}^{y, w}\left(i_{r}^{y, w}\right)$ the oriented arc of the left (right) involute between $y$ and $w$.

Let us introduce now other regions which are bounded by left and right involutes.

Let us fix the initial parameters $x_{0}, s_{0}, \theta_{0}, \vartheta_{0}$.

Definition 28. Given $z \in \mathbb{R}^{2} \backslash K$, let $i_{l}=i_{l, \xi_{l}}\left(i_{r}=i_{r, \xi_{r}}\right)$ be the left (right) involute through $z$ with starting point $\xi_{l}\left(\xi_{r}\right)$ and let $z_{l}\left(z_{r}\right) \in \partial K$ be as in Definition 25. Let $\vartheta_{\xi_{l}}^{+}$satisfying $x_{l}\left(s_{l+}\left(\vartheta_{\xi_{l}}^{+}\right)\right)=\xi_{l}$. Let $\vartheta_{l}>\vartheta_{\xi_{l}}^{+}$be the smallest angle for which $x_{l}\left(s_{l+}\left(\vartheta_{l}\right)\right)=z_{l}$. Let one consider the parameterization (46); let one define

$$
\begin{aligned}
& \mathfrak{G}_{l}(K, z):=\left\{t x_{l}\left(s_{l+}(\vartheta)\right)+(1-t) i_{l}(\vartheta), 0<t\right. \\
& \left.\quad<1, \vartheta_{\xi_{l}}^{+}<\vartheta<\vartheta_{l}\right\} .
\end{aligned}
$$

If $i_{l}^{z}$ does not cross the open segment $z z_{l}$, the region $\mathfrak{G}_{l}(K, z)$ is an open set bounded by the convex $\operatorname{arc}$ of left involute $i_{l}^{z}$, the segment $z z_{l}$, and the convex $\operatorname{arc}$ of $\partial K: \operatorname{arc}^{+}\left(\xi_{l}, z_{l}\right)$; otherwise let $w$ be the nearest point to $z$ where $i_{l}^{z}$ crosses the open segment $z z_{l}$; the region $\mathfrak{G}_{l}(K, z)$ is an open set bounded by the arc $i_{l}^{w, z}$, the segment $w z$, and $\partial K$. Similarly let us define $\mathfrak{G}_{r}(K, z)$. define

$\mathfrak{G}_{l}(K, z), \mathfrak{G}_{r}(K, z)$ are open and bounded sets. Let us

$$
\mathfrak{G}(K, z):=\operatorname{Int}\left(\operatorname{cl}\left(\mathfrak{G}_{l}(K, z) \cup \mathfrak{G}_{r}(K, z)\right)\right) .
$$

$\mathfrak{G}(K, z)$ is an open, bounded, connected set. $\mathfrak{G}(K, z)$ will be called the $\mathfrak{G}$-fence of $K$ at $z$.

Remark 29. If $z$ is the first crossing point of $i_{l}$ and $i_{r}$ and $\xi_{l}=$ $\xi_{r}$, then $\mathfrak{G}(K, z)=\operatorname{Int}\left(\mathfrak{\Im}\left(K, \xi_{l}\right)\right)$.

Let us conclude this section with the following result, which follows from Theorem 20 .

Theorem 30. Let $K$ be limit of a sequence of convex bodies $K^{(n)}, x_{0}=\lim x_{0}^{(n)}$, and $x_{0}^{(n)} \in \partial K^{(n)}$. Then

$$
\mathfrak{J}\left(K^{(n)}, x_{0}^{(n)}\right) \longrightarrow \mathfrak{I}\left(K, x_{0}\right)
$$

Moreover if $z \notin K, z=\lim z^{(n)}, z^{(n)} \notin K^{(n)}$, then

$$
\operatorname{cl}\left(\mathfrak{G}\left(K^{(n)}, z^{(n)}\right)\right) \longrightarrow \operatorname{cl}(\mathfrak{G}(K, z))
$$

\section{Bounding Regions for SDC in the Plane}

Let us assume that $x_{0}$ is the end point of one of the following sets:
(a) a steepest descent curve $\gamma$;
(b) $\gamma^{K}$ : a self-distancing curve from a convex body $K$; see Definition 3.

The following questions arise: can one extend $\gamma, \gamma^{K}$ beyond $x_{0}$ ? Which regions delimit that extension? Which regions are allowed and which are forbidden?

Lemma 31. Let $z \in \mathbb{R}^{2} \backslash K$. If $u \in \mathfrak{G}_{l}(K, z)$ then the arc $i_{l}^{u}$ of the left involute to $K$ ending at $u$ is contained in $\mathfrak{G}_{l}(K, z)$. Similarly if $u \in \mathfrak{G}_{r}(K, z)$, then $i_{r}^{u} \subset \mathfrak{G}_{r}(K, z)$.

Proof. Since $u \in \mathfrak{G}_{l}(K, z)$, by (93) there exist $\bar{\vartheta}_{l} \in\left(\vartheta_{\xi_{l}}^{+}, \vartheta_{l}\right)$, $\tau \in(0,1)$ such that

$$
u=\tau x_{l}\left(s_{l+}\left(\overline{\vartheta_{l}}\right)\right)+(1-\tau) i_{l}\left(\overline{\vartheta_{l}}\right) .
$$

Then the arc $i_{l}^{u}$ is parallel to an arc of the left involute $i_{l}$ (through $z$ ) for $\vartheta \in\left(\vartheta_{\xi_{l}}^{+}, \overline{\vartheta_{l}}\right)$. Then any left tangent segment to $K$ from a point of $i_{l}^{u}$ is contained in the left tangent segment from the corresponding point of $i_{l}^{z}$. 
Lemma 32. Let $z \in \mathbb{R}^{2} \backslash K$ and let $u \in \mathfrak{G}_{l}(K, z)$. There are two possible cases:

(i) if the right involute ending at $u$ does not cross the tangent segment $z_{l} z$ or it crosses $z_{l} z$ at a point $q \epsilon$ $\mathfrak{G}_{l}(K, z)$, then in both cases $i_{r}^{u} \subset \mathfrak{G}_{l}(K, z)$;

(ii) if the right involute ending at $u$ crosses the tangent segment $z_{l} z$ at a point $q \in z_{l} z \cap \partial \mathfrak{G}_{l}(K, z)$, then $i_{r}^{q, u} \backslash\{q\} \subset \mathfrak{G}_{l}(K, z)$.

Proof. Since the starting point $\xi_{r}(u)$ of the right involute ending at $u$ is on $\partial K$, the distance from $\xi_{r}(u)$ to a point of the left involute $i_{l}^{z}$ is not decreasing; see (iv) of Theorem 22; similarly the distance from $\xi_{r}(u)$ to a point of $i_{r}^{u}$ is not decreasing. In case (i) the arc $i_{r}^{u}$ has its end points in $\mathfrak{G}_{l}(K, z)$ and by the above distance property it can not cross two times the left involute; then it can not cross the boundary of $\mathfrak{G}_{l}(K, z)$; therefore $i_{r}^{u} \subset \mathfrak{G}_{l}(K, z)$; similarly in case (ii) the arc $i_{r}^{q, u}$ can not cross the boundary of $\mathfrak{G}_{l}(K, z)$ at most in $q$; therefore all the points of this arc, except to that $q$, belong to $\mathfrak{G}_{l}(K, z)$.

From the previous lemma the following follows.

Theorem 33. Let $z \notin K$. The following inclusions hold:

(a) if $u \in \mathfrak{G}_{l}(K, z)$, then

$$
\operatorname{cl}\left(\mathfrak{G}_{l}(K, u)\right) \backslash \partial K \subset \mathfrak{G}_{l}(K, z) ;
$$

(b) if $u \in \mathfrak{G}_{r}(K, z)$, then

$$
\operatorname{cl}\left(\mathfrak{G}_{r}(K, u)\right) \backslash \partial K \subset \mathfrak{G}_{r}(K, z) ;
$$

(c) if $u \in \mathfrak{G}(K, z)$, then

$$
\operatorname{cl}(\mathfrak{G}(K, u)) \backslash \partial K \subset \mathfrak{G}(K, z) .
$$

Proof. By Lemma 31 the left involute that bounds $\mathfrak{G}_{l}(K, u)$ is inside $\mathfrak{G}_{l}(K, z)$; then $(98)$ is proved. Inclusion (99) is proved similarly. Let $u \in \mathfrak{G}(K, z)=\operatorname{Int}\left(\operatorname{cl}\left(\mathfrak{G}_{l}(K, z) \cup \mathfrak{G}_{r}(K, z)\right)\right)$ and let us consider $u \in \mathfrak{G}_{l}(K, z)$; then in case (i) of Lemma 32 also the open arc of the right involute $i_{r}^{u}$ is inside $\mathfrak{G}_{l}(K, z) \subset$ $\mathfrak{G}(K, z)$. Besides $i_{l}^{u} \subset \partial \mathfrak{G}_{l}(K, u)$; then $(100)$ is trivial. In case (ii) of Lemma 32 the open arc $i_{r}^{q, u}$ is inside $\mathfrak{G}_{l}(K, z)$. On the other hand $q$ is inside $\mathfrak{S}_{r}(K, z)$ and by $(99)$ the $\operatorname{arc} i_{r}^{q} \subset i_{r}^{u}$ is in $\mathfrak{G}_{r}(K, z) \subset \mathfrak{G}(K, z)$. Similar arguments hold if $u \in \mathfrak{G}_{r}(K, z)$. Then (100) holds in this case too.

Lemma 34. Let $w \notin K$. Let $\eta$ be polygonal deleted $S D C_{K}$ with end point $y \in \mathfrak{G}(K, w)$. Then

$$
\begin{aligned}
& \eta \subset \mathfrak{G}(K, w), \\
& \eta \subset \operatorname{cl}(\mathfrak{G}(K, y)) .
\end{aligned}
$$

Proof. To prove (101), let us assume, by contradiction, that $\eta$ has a point $z \notin \mathfrak{G}(K, w)$. With no loss of generality it can be assumed that $z \in \partial \mathfrak{G}(K, w)$ and

$$
\eta \backslash \eta_{z} \subset \mathfrak{G}(K, w) .
$$

Then, $z$ is the end point of a segment $z w_{i}$, where $w_{i} \epsilon$ $\mathfrak{G}(K, w) \cap \eta$ and $z \prec w_{i}$ on $\eta$. As $z \in \partial \mathfrak{G}(K, w)$, then there exists an involute through $z$ which is a piece of the boundary of $\mathfrak{G}(K, w)$ (to fix the ideas it is assumed that it is the left involute $i_{l}$ ). Let us consider $z_{l} \in \partial K$ so that the tangent vector $\mathbf{t}_{z}$ to $i_{l}$ at $z$ satisfies

$$
\left\langle\mathbf{t}_{z}, z-z_{l}\right\rangle=0 .
$$

As $w_{i}$ is inside the orthogonal angle centered in $z$ with sides $\mathbf{t}_{z}$ and $z_{l}-z$, then

$$
\left\langle w_{i}-z, z-z_{l}\right\rangle<0 .
$$

Then as for $\varepsilon>0$ sufficiently small, $z_{\varepsilon}:=z+\varepsilon\left(w_{i}-z\right) \in \eta_{w_{i}}$ and at $z_{\varepsilon}$ the curve $\eta$ has tangent vector $w_{i}-z$ that satisfies

$$
\left\langle w_{i}-z, z_{\varepsilon}-z_{l}\right\rangle<0,
$$

contradicting the fact that $\eta_{w}$ has the distancing from $K$ property (5). This proves (101).

If $w_{n} \rightarrow y$, with $y \in \mathfrak{G}\left(K, w_{n}\right)$, also the inclusions

$$
\eta \subset \operatorname{cl}\left(\mathfrak{G}\left(K, w_{n}\right)\right)
$$

hold. Then (102) is obtained by approximation Theorem 30 .

Theorem 35. Let $K$ be a convex body and let $\gamma^{K}$ be $S D C_{K}$, $w \in \gamma, w \notin K$. Then

$$
\gamma_{w}^{K} \subset \operatorname{cl}(\mathfrak{G}(K, w)) .
$$

Proof. Let us choose a sequence $\left\{w_{n}\right\}, w_{n} \in \gamma^{K}, w_{n} \preceq w, w_{n} \rightarrow$ $w$. Let us fix the $\operatorname{arc} \gamma_{w_{n}}^{K}$. By [8, Theorem 6.16], $\gamma_{w_{n}}^{K}$ is limit of polygonal $\mathrm{SDC}_{K}$ with end point $w_{n}$. From Lemma 34, these polygonal $\mathrm{SDC}_{K}$ are enclosed in $\mathrm{cl}\left(\mathfrak{G}\left(K, w_{n}\right)\right)$; then

$$
\gamma_{w_{n}}^{K} \subset \mathrm{cl}\left(\mathfrak{G}\left(K, w_{n}\right)\right)
$$

holds too. Inclusion (108) is now obtained from the limit of the previous inclusions and by the approximation Theorem 30 .

Theorem 36. Let $K$ be a convex body not reduced to a point. If $\gamma^{K}$ is a self-distancing curve from $K$ with starting point $x_{0} \in$ $\partial K$, then

$$
\gamma^{K} \subset \operatorname{cl}\left(\mathbb{R}^{2} \backslash\left(\mathfrak{\Im}\left(K, x_{0}\right) \cup K\right)\right) .
$$

Proof. Let $z$ be the first crossing point of the left and right involutes of $K$ starting at $x_{0}$. Then

$$
\operatorname{Int}\left(\mathfrak{\Im}\left(K, x_{0}\right)\right)=\mathfrak{G}(K, z) .
$$

By contradiction, if $\gamma^{K}$ has a point $w \in \mathfrak{G}(K, z)$, then, by Theorem 35, the following inclusion holds:

$$
\gamma_{w}^{K} \subset \operatorname{cl}(\mathfrak{G}(K, w)) ;
$$


since, by the distancing from $K$ property, $\gamma^{K}$ has in common with $K$ only the starting point $x_{0}$ then the inclusion

$$
\gamma_{w}^{K} \backslash\left\{x_{0}\right\} \subset \operatorname{cl}(\mathscr{G}(K, w)) \backslash \partial K
$$

holds too. Moreover by (100) the set $\operatorname{cl}(\mathfrak{G}(K, w)) \backslash \partial K$ has positive distance from the $\mathbb{R}^{2} \backslash \mathfrak{G}(K, z)$; then $\gamma_{w}^{K} \backslash\left\{x_{0}\right\}$ has a positive distance from $\mathbb{R}^{2} \backslash \mathfrak{G}(K, z)=\mathbb{R}^{2} \backslash \operatorname{Int}\left(\mathfrak{\Im}\left(K, x_{0}\right)\right)$. This is in contradiction with $x_{0} \in \partial \mathfrak{\Im}\left(K, x_{0}\right)$.

Corollary 37. Let $\gamma$ be SDC and let $z_{1} \in \gamma$; then

$$
\gamma \backslash \gamma_{z_{1}} \subset \operatorname{cl}\left(\mathbb{R}^{2} \backslash \mathfrak{J}\left(\operatorname{co}\left(\gamma_{z_{1}}\right), z_{1}\right)\right)
$$

Proof. Since $\gamma \backslash \gamma_{z_{1}}$ is a self-distancing curve from $\operatorname{co}\left(\gamma_{z_{1}}\right)$ and $z_{1} \in \partial \operatorname{co}\left(\gamma_{z_{1}}\right)($ see $[8,(\mathrm{i})$ of Lemma 4.6]), then Theorem 36 applies to $\gamma^{K}=\gamma \backslash \gamma_{z_{1}}$ with $K=\operatorname{co}\left(\gamma_{z_{1}}\right)$.

Definition 38. Let $\gamma$ be SCD. If $z_{1}, z \in \gamma$, with $z_{1} \preceq z$ let

$$
\gamma_{z_{1}, z}:=\gamma_{z} \backslash \gamma_{z_{1}}
$$

For $z \notin K$, let $K^{z}$ be the cap body, introduced in (9). Next theorem shows the principal result on bounding regions for arcs of SDC $\gamma$.

Theorem 39. Let $K$ be a convex body and let $\gamma$ be $S D C_{K}$. If $z_{1}, z \in \gamma$, with $z_{1} \preceq z$ then

$$
\gamma_{z_{1}, z} \subset \operatorname{cl}\left(\mathfrak{G}(K, z) \backslash \mathfrak{J}\left(K^{z_{1}}, z_{1}\right)\right) .
$$

Proof. First let us notice that $\gamma_{z_{1}, z}$ has the distancing from $K$ and from the set point $\left\{z_{1}\right\}$ property; thus by Proposition 7 it has the distancing from $K^{z_{1}}$ property. Then inclusion (116) follows from Theorems 35 and 36.

Let us conclude the section with the following inclusion result for $\mathfrak{\Im}$-fences.

Theorem 40. Let $K, H$ be two convex bodies not reduced to a point, $K \subset H$. Let $x_{0} \in \partial K \cap \partial H$. Then

$$
\mathfrak{J}\left(K, x_{0}\right) \subset \mathfrak{\Im}\left(H, x_{0}\right) .
$$

Proof. The boundary of $\mathfrak{\Im}\left(H, x_{0}\right)$ consists of two arcs of the left and right involutes of $H$ starting at $x_{0}$. By Corollary 23 they are $\mathrm{SDC}_{H}$, and then they are $\mathrm{SDC}_{K}$; therefore by Theorem 36 they cannot intersect the boundary of $\mathfrak{\Im}\left(K, x_{0}\right)$.

4.1. Minimally Connecting Plane Steepest Descent Curves. Given a point $x_{1} \notin K$, the segment joining it with its projection $x_{0}$ on $\partial K$ is $\mathrm{SDC}_{K}$ which minimally connects the two points.

This subsection is devoted to consider when it would be possible to connect a given point $x_{0}$ on the boundary of a plane convex body $K$, with an arbitrarily given point $x_{1} \notin K$, by using a steepest descent curve $\gamma \in$ SDC $_{K}$. Let us denote with $\Gamma_{x_{0}, x_{1}}^{K}$ the class of the curves $\gamma \in \operatorname{SDC}_{K}$ starting at $x_{0}$ and ending at $x_{1}$.

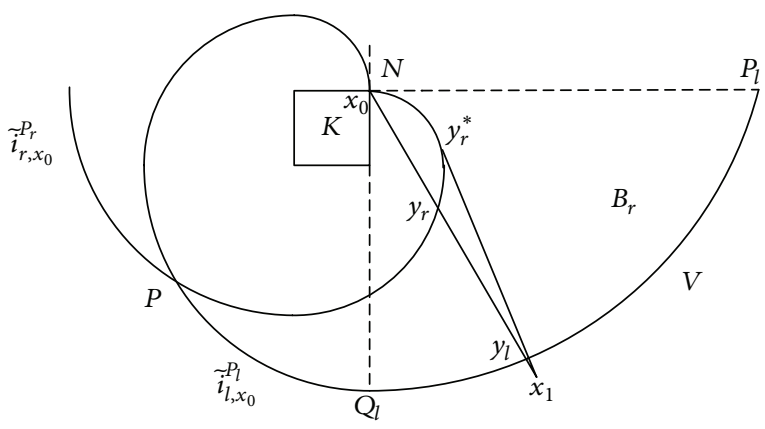

FIgURE 4: The regions $N, B_{r}$, and $V$ when $K$ is a square.

Definition 41. Let $\gamma$ be SDC with end point $y$ and let $\eta$ be SDC with starting point $y$; let us denote by $\gamma * \eta$ the curve joining $\gamma$ with $\eta$ in the natural order, if it is SDC curve.

Theorem 42. Let $x_{0} \in \partial K, x_{1} \notin K$. Then $\Gamma_{x_{0}, x_{1}}^{K} \neq \emptyset$ iff

$$
x_{1} \in \operatorname{cl}\left(\mathbb{R}^{2} \backslash\left(\mathfrak{\Im}\left(K, x_{0}\right)\right) \cup K\right)
$$

If (118) holds, there exist at most two $\eta_{i} \in \Gamma_{x_{0}, x_{1}}^{K}, i=1,2$ such that the following properties are true:

$$
\begin{gathered}
\forall \gamma \in \Gamma_{x_{0}, x_{1}}^{K} \Longrightarrow \\
\operatorname{co}\left(\eta_{1}\right) \subset \operatorname{co}(\gamma) \\
\text { or } \operatorname{co}\left(\eta_{2}\right) \subset \operatorname{co}(\gamma),(\text { or both }), \\
\forall \gamma \in \Gamma_{x_{0}, x_{1}}^{K} \Longrightarrow \\
|\gamma| \geq \min _{i=1,2}\left\{\left|\eta_{i}\right|\right\}
\end{gathered}
$$

Proof. Let $\gamma \in \Gamma_{x_{0}, x_{1}}^{K}$. From (110) of Theorem 36, since $x_{1} \in \gamma$, then (118) follows.

Let us prove now that (118) is sufficient. Let us notice that $\mathbb{R}^{2} \backslash\left(\mathfrak{\Im}\left(K, x_{0}\right) \cup K\right)$ can be divided into four regions $N, B_{l}$, $B_{r}$, and $V$ (see Figure 4 ) defined as follows:

(i) the closed normal sector $N:=x_{0}+N_{K}\left(x_{0}\right)$ is the angle bounded by the two half lines $t_{l}, t_{r}$ tangent at $x_{0} \in$ $\partial K$ to the left and right involute $i_{l}:=i_{l, x_{0}}, i_{r}:=i_{r, x_{0}}$, respectively; this angle can be reduced to an half line, starting at $x_{0}$;

(ii) let $P$ be the first crossing point between $i_{l}$ and $i_{r}$; see Theorem $24 ; i_{l}$ is SDC to $i_{l}\left(\vartheta_{l}^{*}\right)$, which will be a point $Q_{l}$ following $P$; after $Q_{l}$ the involute $i_{l}$ is no more SDC; see (i) of Theorem 22.

Let us change $i_{l}$ after $Q_{l}$ with $j_{l, Q_{l}}$, the left involute of $\operatorname{co}\left(K \cup i_{l}^{Q_{l}}\right)$ at $Q_{l}$.

Let us define $P_{l}$ as the first intersection point of $j_{l, \mathrm{Q}_{l}}$ with $\partial N$, and let

$$
\stackrel{P}{i}_{l, x_{0}}^{P_{l}}:=i_{l, x_{0}}^{\mathrm{Q}_{l}} * j_{l, \mathrm{Q}_{l}}^{P_{l}}
$$


It is not difficult to see that $\tilde{i}_{l, x_{0}} \in \Gamma_{x_{0}, P_{l}}^{K}$. Changing the left with the right, $\widetilde{i}_{r, x_{0}}$ and the point $P_{r}$ can be constructed. Let $B_{r}$ be the union of the $\operatorname{arc} i_{r, x_{0}}^{P} \backslash\{P\}$ with the plane open region bounded by the segment $x_{0} P_{l}$, the $\operatorname{arc} i_{r, x_{0}}^{P}$, and the $\operatorname{arc} \tilde{i}_{l}^{P, P_{l}}$; let $B_{l}$ be the union of the arc $i_{l, x_{0}}^{P} \backslash\{P\}$ with the plane open region bounded by the segment $x_{0} P_{r}$, the $\operatorname{arc} i_{l, x_{0}}^{P}$, and the arc $\stackrel{i}{r}_{r}^{P, P_{r}}$;

(iii) let $V$ be the remaining region; that is, $V=\mathbb{R}^{2} \backslash(K \cup$ $\left.\mathfrak{\Im}\left(K, x_{0}\right) \cup N \cup B_{l} \cup B_{r}\right)$.

Let $x_{1} \in B_{r} \cup V$. On the oriented curve $\widetilde{i}_{r, x_{0}}^{P_{r}}$ there are two points so that their tangent lines contain $x_{1}$. Let $y_{r}^{*}$ be the first tangency point.

Let $y_{l}, y_{r}$ be the intersection points of the half line $m$ starting at $x_{0}$ and containing $x_{1}$, with $\widetilde{i}_{l, x_{0}}$ and with $\tilde{i}_{r, x_{0}}$, respectively; see Figure 4.

Under assumption (118), $x_{1}$ belongs to one of the four regions $N, B_{l}, B_{r}, V$; let us prove now (119), (120) in the four corresponding cases.

(1) If $x_{1} \in N$, then let $\eta_{1}=\eta_{2} \in \Gamma_{x_{0}, x_{1}}^{K}$ be the segment $x_{0} x_{1}$. Then (119) and (120) are trivial.

(2) Let $x_{1} \in V$. Let

$$
\eta_{r}:=\widetilde{i}_{r, x_{0}}^{y_{0}^{*}} * y_{r}^{*} x_{1} .
$$

The curve $\eta_{r}$ is $\mathrm{SDC}_{K}$, since the normal lines at all the points on the segment $y_{r}^{*} x_{1}$ have the same directions and support $\hat{i}_{r, x_{0}}^{\sim_{r}^{*}}$ up to $y_{r}^{*}$; then $\eta_{r}$ is $\mathrm{SDC}_{K}$ and joins $x_{0}$ with $x_{1}$. Similarly $\mathrm{SDC}_{K}$ is defined:

$$
\eta_{l}=\widetilde{i}_{l, x_{0}}^{y_{l}^{*}} * y_{l}^{*} x_{1} .
$$

Thus $\Gamma_{x_{0}, x_{1}}^{K}$ is nonempty and contains at least the two elements $\eta_{l}, \eta_{r}$.

Let us consider the connected closed curve

$$
c_{x_{1}}:=\widetilde{i}_{r, x_{0}}^{y_{r}} \cup y_{r} y_{l} \cup \widetilde{i}_{l, x_{0}}^{y_{l}} .
$$

Let $\gamma \in \Gamma_{x_{0}, x_{1}}^{K}$, and let $T \ni t \rightarrow x(t) \in \gamma$ be a continuous parameterization of $\gamma$. Let us project from $x_{0}$ the curve $\gamma$ on $c_{x_{1}}$ and let $D$ be this projection. That is, for $t \in T$, let $\lambda_{t}:=\left\{x_{0}+\lambda x(t), 0 \leq \lambda\right\}$ and let

$$
D=\bigcup_{t \in T}\left(c_{x_{1}} \cap \lambda_{t}\right) \text {. }
$$

Clearly $D$ is a closed connected subset of $c_{x_{1}}$ containing $x_{0}$ and the segment $y_{r} y_{l}$. Thus $D$ contains at least one of the two connected components of $c_{x_{1}}$ joining $x_{0}$ with $y_{r}, y_{l}$. Therefore the inclusions

$$
\left(\tilde{i}_{r, x_{0}}^{y_{r}} \cup y_{r} y_{l}\right) \subset D
$$

or

$$
\left(\widetilde{i}_{l, x_{0}}^{y_{l}} \cup y_{r} y_{l}\right) \subset D
$$

(or both) hold.

Assume that (126) holds and let $\eta_{r}$ be defined as in (122). Since, by construction of $D$, the set $\operatorname{co}\left(D \cup\left\{x_{1}\right\}\right)$ is contained in $\operatorname{co}(\gamma)$, then

$$
\operatorname{co}\left(\eta_{r}\right) \subset \operatorname{co}(\gamma) \text {. }
$$

Similarly if (127) holds, then

$$
\operatorname{co}\left(\eta_{l}\right) \subset \operatorname{co}(\gamma),
$$

with $\eta_{l}$ defined by (123). Then (119) is proved. It is not difficult to see that the region bounded by $\gamma \cup x_{0} x_{1}$ contains the convex region bounded by $i_{r, x_{0}}^{y_{r}^{*}} \cup y_{r}^{*} x_{1} \cup$ $x_{0} x_{1}$. Thus the bound

$$
|\gamma| \geq\left|i_{r, x_{0}}^{y_{r}^{*}}\right|+\left|y_{r}^{*} x_{1}\right|
$$

holds; similar procedure can be used for left case. This proves (120).

(3) Let $x_{1} \in B_{r}$; the same argument as in case (2) can be carried on up to the inclusions (126) and (127). As in step (2), when case (126) holds, the curve $\eta_{r}$ can be constructed and $\eta_{r}$ is $\mathrm{SDC}_{K}$.

Let us show that if $x_{1} \in B_{r}$ then (127) cannot occur, so the curve $\eta_{l}$ can not to be constructed.

Let us argue by contradiction. If (127) occurs, then let $z_{1} \neq x_{0}$ be the first point where $\gamma$ crosses the half line $x_{0} P$. The point $z_{1}$ exists, since, under the assumption (127), $P \in D$. Then (Theorem 36) $z_{1}$ does not belong to the open segment $x_{0} P$. Then, from (127) (see Definition 27)

$$
\operatorname{co}\left(\gamma_{z_{1}}\right) \supset \mathfrak{\Im}_{l}\left(K, x_{0}\right) .
$$

Moreover $\gamma \backslash \gamma_{z_{1}} \cup\left\{z_{1}\right\}$ is $\mathrm{SDC}_{\mathrm{co}\left(K \cup \gamma_{z_{1}}\right)}$; see Remark 6 . Then by (131) it is $\mathrm{SDC}_{\mathrm{co}\left(K \cup \widetilde{\mathfrak{I}}_{l}\left(K, x_{0}\right)\right) \text {. Let us consider }}$ the convex body $H=\operatorname{co}\left(K \cup i_{l, x_{0}}^{P}\right)$. Since

$$
\operatorname{co}\left(K \cup \gamma_{z_{1}}\right) \supset \operatorname{co}\left(K \cup \mathfrak{\Im}_{l}\left(K, x_{0}\right)\right) \supset H,
$$

then $\gamma \backslash \gamma_{z_{1}}$ is a deleted $\mathrm{SDC}_{H}$. From Theorem 39, with $H$ in place of $K, x_{1}$ in place of $z$, it follows that

$$
\gamma_{z_{1}, x_{1}} \subset \operatorname{cl}\left(\mathbb{R}^{2} \backslash \mathfrak{I}\left(H^{z_{1}}, z_{1}\right)\right) .
$$

Let $P_{1}$ be where the right tangent from $z_{1}$ to $H$ crosses the arc $\tilde{i}_{l, P}$. Let us notice that $\ddot{i}_{l, P}$ is also an arc of the left involute of $H$ at $P$. Moreover ${ }_{i}^{P_{1}}, P_{l}$ is an arc of the left involute of $H^{z_{1}}$ starting at $P_{1}$; then it is parallel to the left involute of $H^{z_{1}}$ at $z_{1}$ until it crosses the sector $N$; it turns out that

$$
B_{r} \subset \mathfrak{J}\left(H^{z_{1}}, z_{1}\right) .
$$


From the two previous inclusions a contradiction comes out since

$$
x_{1} \in \gamma_{z_{1}, x_{1}} \cap B_{r}=\emptyset .
$$

Then (127) cannot occur.

(4) The case $x_{1} \in B_{l}$ is similar to the previous one.

The proof is complete.

Definition 43. Under the assumptions of Theorem 42, let one define $E_{x_{0}, x_{1}}^{K}$ the set of $\eta_{i}, i=1,2$ (possibly coinciding) as they are constructed in the proof of Theorem 42, which satisfy (119) and (120). These curves will be called minimally connecting steepest descent curves for the class $\Gamma_{x_{0}, x_{1}}^{K}$.

Definition 44. Let $\gamma: T \ni t \rightarrow x(t)$ be an absolutely continuous curve and let $x(t)$ be a point of $\gamma$, with tangent vector $\dot{x}(t)$. Let

$$
\mathscr{H}_{x(t)}:=\left\{y \in \mathbb{R}^{2}:\langle\dot{x}(t), y-x(t)\rangle \leq 0\right\} .
$$

$\mathscr{H}_{x(t)}$ is half plane bounded by the normal line to $\gamma$ at $x(t)$ and it is defined almost everywhere in T. For the curve $\gamma \backslash \gamma_{x_{1}}$ (consisting of the points of $\gamma$ following $x_{1}$ ) let us define the region:

$$
\mathfrak{H}\left(\gamma, x_{1}\right):=\bigcap_{x_{1} \leq x(t), x(t) \in \gamma} \mathscr{H}_{x(t)}
$$

If $\mathfrak{S}\left(\gamma, x_{1}\right) \neq \emptyset$, then it is a convex set.

If $\gamma$ is $\operatorname{SDC}\left(\gamma\right.$ is $\left.\operatorname{SDC}_{K}\right)$, then condition (2) (resp., (5)) implies that

$$
\left.\gamma_{x_{1}} \subset \mathfrak{H}\left(\gamma, x_{1}\right) \quad \text { (resp., } \gamma_{x_{1}} \cup K \subset \mathfrak{H}\left(\gamma, x_{1}\right)\right) .
$$

Theorem 45. Let $x_{0} \in \partial K, x_{1} \notin K$. Let $\gamma_{1}$ be SDC with first point $x_{1}$. Necessary and sufficient conditions for the existence of a curve $\gamma$, self-distancing curve from $K$, starting at $x_{0}$ and satisfying $\left(\gamma \backslash \gamma_{x_{1}}\right) \cup\left\{x_{1}\right\}=\gamma_{1}$, are as follows:

(a) $x_{1} \in \operatorname{cl}\left(\mathbb{R}^{2} \backslash \mathfrak{\Im}\left(K, x_{0}\right)\right)$;

(b) there exists $\eta \in E_{x_{0}, x_{1}}^{K}$ such that $(K \cup \eta) \subset \mathfrak{H}\left(\gamma_{1}, x_{1}\right)$; moreover if (a) and (b) are satisfied, then $\gamma_{1} \in S D C_{\mathrm{co}(K \cup \eta)}$.

Proof. (a) is necessary by Theorem 36. (b) is necessary by Theorem 42 and by (138) since $\mathfrak{S}\left(\gamma_{1}, x_{1}\right)=\mathfrak{S}\left(\gamma, x_{1}\right)$. Conversely if (a) and (b) hold, let us define $\gamma:=\eta * \gamma_{1}$; then (by definition of $\left.E_{x_{0}, x_{1}}^{K}\right) \eta$ is $\mathrm{SDC}_{K}$; thus, $\gamma$ is $\mathrm{SDC}_{K}$ too and $\gamma_{1}$ is $\mathrm{SDC}_{\mathrm{co}(K \cup \eta)}$ (see Remark 6).

\section{Self-Distancing Sets and Steepest Descent Curves}

A self-distancing set $\sigma$ will be called SDC-extendible if there exists a steepest descent curve $\gamma$ such that $\sigma \subset \gamma$.
This section is devoted to investigate the following question:

Can a self-distancing set $\sigma$ be extended to a steepest descent curve $\gamma$ ?

Let us call $\Gamma_{\sigma}$ the family of SDC $\gamma$ which extends $\sigma$. The following example shows that $\Gamma_{\sigma}$ can be empty.

Example 46. Let us consider in a coordinate system $x y$ the points

$$
\begin{aligned}
& x_{1}=(0,0), \\
& x_{2}=(0,2), \\
& x_{3}=(1, \sqrt{8}), \\
& x_{4}=(-1, \sqrt{8}) .
\end{aligned}
$$

The set $\widetilde{\sigma}=\left\{x_{i}, i=1, \ldots, 4\right\}$ is a self-distancing set not SDCextendible.

Proof. By contradiction let $\gamma \in \Gamma_{\widetilde{\sigma}}$; then any point $x$ on the arc $\gamma_{x_{3}, x_{4}}$ satisfies the inequalities

$$
\begin{aligned}
3 & =\left|x_{3}-x_{1}\right| \leq\left|x-x_{1}\right| \leq\left|x_{4}-x_{1}\right|=3, \\
\left|x_{3}-x_{2}\right| & \leq\left|x-x_{2}\right| .
\end{aligned}
$$

That is, $x \in \partial B\left(x_{1}, 3\right)$ and $x \in \mathbb{R}^{2} \backslash B\left(x_{2},\left|x_{3}-x_{2}\right|\right)$. Since $\partial B\left(x_{1}, 3\right) \cap\left(\mathbb{R}^{2} \backslash B\left(x_{2},\left|x_{3}-x_{2}\right|\right)\right)=\left\{x_{3}, x_{4}\right\}$, the arc $\gamma_{x_{3}, x_{4}}$ consists of two points only, which is impossible.

Next theorem gives a necessary condition (141) in order to extend a finite self-distancing set $\sigma$ to SDC; this condition is based on the bounding sets introduced in Section 3.1.

Let us define $\sigma_{x}$ as the subset of $\sigma$ consisting of the point $x$ and of the previous ones on $\sigma$ (consistent with (3)).

Theorem 47. Let $\sigma$ be a self-expanding SDC-extendible set; then for all $x_{0} \in \sigma$ such that $\sigma_{x_{0}} \neq\left\{x_{0}\right\}$, the inclusion

$$
\left(\sigma \backslash \sigma_{x_{0}}\right) \subset \operatorname{cl}\left(\mathbb{R}^{2} \backslash \mathfrak{\Im}\left(\operatorname{co}\left(\sigma_{x_{0}}\right), x_{0}\right)\right)
$$

holds.

Proof. Let $\gamma \in \Gamma_{\sigma}$; then $\sigma \subset \gamma$ and $\gamma$ is SDC. Then $\operatorname{co}\left(\gamma_{x_{0}}\right) \supset \operatorname{co}\left(\sigma_{x_{0}}\right)$ and $x_{0} \in \partial \operatorname{co}\left(\gamma_{x_{0}}\right) \cap \partial \operatorname{co}\left(\sigma_{x_{0}}\right)$ (see [8, (i) of Lemma 4.6]); from Theorem 40,

$$
\mathfrak{J}\left(\operatorname{co}\left(\gamma_{x_{0}}\right), x_{0}\right) \supset \mathfrak{I}\left(\operatorname{co}\left(\sigma_{x_{0}}\right), x_{0}\right) ;
$$

moreover $\sigma \backslash \sigma_{x_{0}} \subset \gamma \backslash \gamma_{x_{0}}$ and from Corollary 37,

$$
\gamma \backslash \gamma_{x_{0}} \subset \operatorname{cl}\left(\mathbb{R}^{2} \backslash \mathfrak{J}\left(\operatorname{co}\left(\gamma_{x_{0}}\right), x_{0}\right)\right) \text {. }
$$

The previous inclusions prove (141).

Remark 48. In Example 46 it has been proved, in a simple way, that $\widetilde{\sigma}$ is not SDC-extendible. Another way to prove this 
fact is to check that condition (141) does not hold for the point $x_{4}$; let us notice that $\partial \mathfrak{J}\left(\operatorname{co}\left(\widetilde{\sigma}_{x_{3}}\right), x_{3}\right) \cap\{x \leq 0, y \geq 0\}$ consists of a circular arc centered at $x_{1}$ with radius $2+\sqrt{13-4 \sqrt{8}}$; then it is easy to see that $x_{4}$ is in the interior of $\mathfrak{J}\left(\operatorname{co}\left(\widetilde{\sigma}_{x_{3}}\right), x_{3}\right)$ and (141) is not satisfied.

Let us show in the following example that (141) is not sufficient for a self-distancing set $\sigma$ to be SDC-extendible.

Example 49. Let us consider in a coordinate system $x y$ the points

$$
\begin{aligned}
& \xi_{1}=(0,0), \\
& \xi_{2}=(0,2), \\
& \xi_{3}=(2,0), \\
& \xi_{4}=(\rho, 2) .
\end{aligned}
$$

For $\sqrt{8}<\rho<\pi$, the set $\sigma:=\left\{\xi_{i}, i=1, \ldots, 4\right\}$ is a selfdistancing set satisfying condition (141) not SDC-extendible.

Proof. It easy to see that $\sigma$ is a self-distancing set. Moreover the initial piece of the left involute of $\operatorname{co}\left(\left\{\xi_{1}, \xi_{2}, \xi_{3}\right\}\right)$ starting at $\xi_{3}$ consists of a circular arc centered at $\xi_{2}$ of ray $\sqrt{8}$ and amplitude $(3 / 4) \pi$. Then $\xi_{4} \notin \mathfrak{J}\left(\operatorname{co}\left(\left\{\xi_{1}, \xi_{2}, \xi_{3}\right\}\right), \xi_{3}\right)$ and (141) is verified with $x_{0}=\xi_{3}, \sigma \backslash \sigma_{x_{0}}=\left\{\xi_{4}\right\}$. Trivially (141) is verified also at $x_{0}=\xi_{2}$. Let us prove now that $\Gamma_{\sigma}$ is empty. By contradiction let $\gamma \in \Gamma_{\sigma}$. Let us consider $\gamma_{\xi_{3}}$. Since $\xi_{2}$, $\xi_{3}$ have the same distance from $\xi_{1}$, arguing as in Example 46, $\gamma_{\xi_{3}}$ is a circular arc $C$ centered at $\xi_{1}$ from $\xi_{2}$ to $\xi_{3}$. Since the arc $\gamma_{\xi_{3}}$ has the distancing property from the segment $\xi_{1} \xi_{2}$, it is necessarily the arc of amplitude $\pi / 4$ and not the complementary arc. Let $\eta=\xi_{1} \xi_{2} * C$. Since $C \subset \gamma$ and $\xi_{2}, \xi_{3} \in \gamma$, then $\operatorname{co}(\eta) \subset \operatorname{co}\left(\gamma_{\xi_{3}}\right)$. Thus by Theorem 40

$$
\mathfrak{J}\left(\operatorname{co}\left(\gamma_{\xi_{3}}\right), \xi_{3}\right) \supset \mathfrak{I}\left(\operatorname{co}(\eta), \xi_{3}\right) .
$$

Since the segment $\xi_{2} \xi_{4}$ is tangent to $\eta$ at $\xi_{2}$ and it has length $\rho$, less than $\pi$, the length of the $\operatorname{arc} \eta_{\xi_{2}, \xi_{3}}$, then

$$
\xi_{4} \in \operatorname{Int}\left(\mathfrak{J}\left(\operatorname{co}\left(\gamma_{\xi_{3}}\right), \xi_{3}\right)\right) .
$$

This is in contradiction with Corollary 37 at the point $\xi_{3}$.

Let us introduce definitions and preliminary facts needed to obtain necessary and sufficient conditions for the extendibility of a self-distancing set $\sigma$ structured as follows.

Definition 50. Let one denote with $\widetilde{\cup}_{i} \sigma_{i}$ a self-distancing set with a finite (or countable) family of closed connected components $\sigma_{1}, \sigma_{2}, \ldots, \sigma_{n}, \ldots$, ordered as the points of $\sigma$; that is, if $i<j, x \in \sigma_{i}, y \in \sigma_{j} \Rightarrow x \leq y$. Let $x_{i}^{-}$be the first point and let $x_{i}^{+}$be the last point of $\sigma_{i}$; if they are distinct (i.e. $\sigma_{i}$ does not reduce to a point) as noticed in the introduction ([6, Theorem 3.3] and [8, Theorem 4.10]), $\sigma_{i}$ is SDC and it will be denoted by $\gamma_{i}$.
Lemma 51. Let $\sigma=\widetilde{\cup}_{i} \sigma_{i}$ be a self-distancing set. A necessary condition for $\Gamma_{\sigma} \neq \emptyset$ is that for all components $\sigma_{i}$, which are curves $\gamma_{i}$, the fact

$$
\bigcup_{j=1}^{i} \sigma_{j} \subset \mathfrak{H}\left(\gamma_{i+1}, x_{i+1}^{-}\right)
$$

holds.

Proof. Let $\gamma \in \Gamma_{\sigma}$. Then $\cup_{j=1}^{i} \sigma_{j} \subset \gamma_{x_{i+1}^{-}}$. Then (147) follows from (138).

Definition 52. Let $\sigma=\widetilde{\mathrm{U}}_{i} \sigma_{i}$ be a self-distancing set. A subfamily $E_{\sigma} \subset \Gamma_{\sigma}$ is called essential for $\Gamma_{\sigma}$ if the facts

$$
\begin{array}{ll}
\text { (a) } & \forall \gamma \in \Gamma_{\sigma} \exists \eta \in E_{\sigma}: \operatorname{co}(\eta) \subset \operatorname{co}(\gamma), \\
\text { (b) } & \gamma \in \Gamma_{\sigma} \Longrightarrow \\
& |\gamma| \geq \min \left\{|\eta|, \eta \in E_{\sigma}\right\}, \\
\text { (c) } & \gamma \in \Gamma_{\sigma},|\gamma|=\min \left\{|\eta|, \eta \in E_{\sigma}\right\} \Longrightarrow \\
& \gamma \in E_{\sigma}
\end{array}
$$

hold.

If $\Gamma_{\sigma}=\emptyset$, let us define $E_{\sigma}=\emptyset$ essential for $\Gamma_{\sigma}$.

Let us start to study a self-distancing set with two closed connected components.

Lemma 53. Let $\sigma^{*}=\widetilde{\mathrm{U}}_{i=1,2} \sigma_{i}$ be a self-distancing set and let $\rho$ be the segment joining $x_{1}^{+}, x_{2}^{-}$. There are five possibilities:

(p1) Let $\sigma_{1}=\left\{x_{1}\right\}, \sigma_{2}=\left\{x_{2}\right\}$; then $E_{\sigma^{*}}=\{\rho\} \neq \emptyset$ is essential for $\Gamma_{\sigma}$.

(p2) Let $\sigma_{1}=\left\{x_{1}\right\}, \sigma_{2}=\gamma_{2}\left(\gamma_{2}\right.$ is SDC); then a necessary and sufficient condition for the extensibility of $\sigma^{*}$ is

$$
\sigma_{1} \subset \mathfrak{H}\left(\gamma_{2}, x_{2}^{-}\right) ;
$$

moreover $E_{\sigma^{*}}=\left\{\rho * \gamma_{2}\right\}$ is essential for $\Gamma_{\sigma^{*}}$.

(p3) Let $\sigma_{1}=\gamma_{1}$ be a SDC, $\sigma_{2}=\left\{x_{2}\right\}$; then a necessary and sufficient condition for the extensibility of $\sigma^{*}$ is

$\sigma_{2} \subset \operatorname{cl}\left(\mathbb{R}^{2} \backslash\left(\mathfrak{J}\left(\operatorname{co}\left(\gamma_{1}\right), x_{1}^{+}\right)\right) \cup \operatorname{co}\left(\gamma_{1}\right)\right) ;$

moreover $E_{\sigma^{*}}=\left\{\gamma_{1} * \eta: \eta \in E_{x_{1}^{+}, x_{2}}^{\mathrm{co}\left(\gamma_{1}\right)}\right\}$ (see Definition 43).

(p4) Let $\sigma_{1}=\gamma_{1}, \sigma_{2}=\gamma_{2}$; then a necessary and sufficient condition for the extensibility of $\sigma^{*}$ is that there exists SDC $\eta$ such that

$$
\begin{gathered}
\eta \in E_{x_{1}^{+}, x_{2}^{-}}^{\operatorname{co}\left(\gamma_{1}\right)}, \\
\sigma_{1} \cup \eta \subset \mathfrak{H}\left(\gamma_{2}, x_{2}^{-}\right) ;
\end{gathered}
$$

the related essential family is $E_{\sigma^{*}}=\left\{\gamma_{1} * \eta * \gamma_{2}: \eta\right.$ satisfies (153)\}. 
(p5) If in cases (p2), ( $p 3)$, and ( $p 4)$ the corresponding necessary and sufficient conditions do not hold, then

$$
E_{\sigma^{*}}=\Gamma_{\sigma^{*}}=\emptyset \text {. }
$$

Proof. Case (p1) is trivial; in case (p2) the inclusion (151) follows from Lemma 51 with $i=2$ in (147). It is also trivial that it is sufficient. Case (p3) follows from Theorem 42 with $K=\operatorname{co}\left(\gamma_{1}\right)$. Case $(\mathrm{p} 4)$ follows from Theorem 45 with $\gamma_{2}$ in place of $\gamma_{1}, K=\operatorname{co}\left(\gamma_{1}\right), x_{1}^{+}$in place of $x_{0}$ and $x_{2}^{-}$in place of $x_{1}$.

An easy sufficient condition to check if $\sigma=\widetilde{U}_{i} \sigma_{i}$ is extendible is the following.

Theorem 54. Let $\sigma=\widetilde{\cup}_{i} \sigma_{i}$ be a self-distancing set. Let $\sigma^{(i)}=$ $\tilde{\mathrm{u}}_{j=1}^{i} \sigma_{j}$. If (147) and

$$
x_{i+1}^{-} \in N_{\mathrm{co}\left(\sigma^{(i)}\right)}\left(x_{i}^{+}\right), \quad \forall i \geq 1
$$

hold, then $\Gamma_{\sigma} \neq \emptyset$ and $\bar{\gamma}$, which linearly and orderly connects $\sigma_{i}, \sigma_{i+1}$ with the segments $x_{i}^{+} x_{i+1}^{-}$, is SDC and it has minimal length in $\Gamma_{\sigma}$.

Proof. Let us argue by induction on the self-distancing set $\sigma^{(i)}$. The case $i=1$ is contained in Lemma 53, since the assumptions (147) and (155) are enough to get the corresponding assumptions in cases (p1), (p2), (p3), and (p4). Moreover in the case $(\mathrm{p} 4)$ the curve $\eta=x_{1}^{+} x_{2}^{-}$is such that $\gamma^{(2)}=\gamma_{1} * \eta * \gamma_{2}$ is the SDC of minimal length extending $\sigma^{(2)}$.

Let $\gamma^{(i)}$ be the curve of minimal length extending $\sigma^{(i)}$. Since the normal sector to $\operatorname{co}\left(\sigma^{(i)}\right)$ at $x_{i}^{+}$coincides with the sector $N$ in the proof of Theorem 42, with $x_{i}^{+}$in place of $x_{0}$, $x_{i+1}^{-}$in place of $x_{1}$, then assumption (155) implies that case (1) of the proof of Theorem 42 occurs. It follows that

$$
\gamma^{(i+1)}=\gamma^{(i)} * x_{i}^{+} x_{i+1}^{-} * \sigma_{i+1}
$$

is of minimal length in $\Gamma_{\sigma^{(i+1)}}$. Then, $\bar{\gamma}=\cup_{i} \gamma^{(i+1)}$ is of minimal length in $\Gamma_{\sigma}$.

Remark 55. Since, as noticed in [4, II, Section 2], $\forall u, w \in \sigma_{x_{i}^{+}}$ the angle $u x_{i}^{+} w$ has opening less than $\pi / 2$, thus the related normal sector in (155) has opening greater than or equal to $\pi / 2$; then checking that $x_{i+1}^{-}$satisfies (155) is easier than checking that $x_{i+1}^{-}$is outside of the $\mathfrak{F}$-fence as in (152).

Lemma 51 and Theorem 54 give only necessary and only sufficient conditions, respectively, for the extensibility of self-expanding sets. Let us give definitions in order to get necessary and sufficient conditions.

Definition 56. Let $\sigma=\widetilde{U}_{j} \sigma_{j}$ be a self-distancing set. Let $E_{i}, i=$ $2, \ldots, n, \ldots$ be defined by induction as follows.

$E_{2}$ is the essential family related to $\tilde{\cup}_{j=1}^{2} \sigma_{j}$, as given by Lemma 53; if $i \geq 2$, the $E_{i+1}$ related to $\tilde{\cup}_{j=1}^{i+1} \sigma_{j}$ is defined as follows:

(i) if $E_{i}=\emptyset$ then $E_{i+1}=\emptyset$; (ii) if $E_{i} \neq \emptyset$, let one consider for all $\eta \in E_{i}$ the essential family $E(\eta)$ (see Lemma 53) related to $\eta \widetilde{\cup} \sigma_{i+i}$ (see Definition 50). Let $E_{i+1}=\cup_{\eta \in E_{i}} E(\eta)$.

Let us notice that $\left\{E_{i}\right\}$ is ordered by inclusion and $E_{i+1}$, if it is nonempty, consists of $2^{i}$ curves at most.

Theorem 57. Let $\sigma=\tilde{U} \sigma_{j}$ be a self-expanding set and let $E_{2}, E_{3}, \ldots, E_{i}, \ldots$ be the sequence (finite or countable) of the essential families associated with $\sigma$. Then $\Gamma_{\sigma} \neq \emptyset$ iff $\forall i \geq 2$ the essential family $E_{i}$ is nonempty.

Proof. If there exists $\gamma \in \Gamma_{\sigma}$ then, for all $i \geq 1, \gamma_{x_{i+1}^{+}} \in \Gamma_{\widetilde{\cup}_{j=1}^{i+1} \sigma_{j}}$; thus $E_{i+1} \neq \emptyset$ by Theorem 42 . Conversely if at each step $i \geq 1$ the essential family $E_{i+1} \neq \emptyset$, then, by definition, there exists a sequence $\left\{\eta^{i+1}\right\}$ of SDC such that $\eta^{i+1} \in E_{i+1}$ and such that $\eta^{s} \subset \eta^{s+1}, s \geq 1$ (i.e., at each step, $\eta^{s+1}$ is an extension of a previous one $\eta^{s}$ ) and $\eta^{s+1} \in E_{\widetilde{\cup}_{j=1}^{i+1} \sigma_{j}}$; see Definition 56. Then $\gamma=\cup_{i=1}^{\infty} \eta^{i+1}$ is well defined; obviously $\gamma \in \Gamma_{\sigma}$.

Open Problem. In the present work only two-dimensional problems are studied. In three (or more) dimensions the construction of boundary regions to $\mathrm{SDC}$ and to $\mathrm{SDC}_{K}$ is open. The boundary regions should probably be constructed by using the space involutes of the geodesics curves on $\partial K$.

\section{Competing Interests}

The authors declare that they have no competing interests.

\section{Acknowledgments}

This work has been partially supported by INdAM-GNAMPA (2014).

\section{References}

[1] W. Fenchel, Convex Cones, Sets and Functions, Princeton University Press, Princeton, NJ, USA, 1953.

[2] C. Icking, R. Klein, and E. Langetepe, "Self-approaching curves," Mathematical Proceedings of the Cambridge Philosophical Society, vol. 125, no. 3, pp. 441-453, 1999.

[3] I. F. Mainnik, "An estimate of length for the curve of descent," Sibirskiu Matematicheskiu Zhurnal, vol. 33, no. 4, pp. 215-218, 1992.

[4] P. Manselli and C. Pucci, "Maximum length of steepest descent curves for quasi-convex functions," Geometriae Dedicata, vol. 38, no. 2, pp. 211-227, 1991.

[5] J. Bolte, A. Daniilidis, O. Ley, and L. Mazet, "Characterizations of Łojasiewicz inequalities: subgradient flows, talweg, convexity," Transactions of the American Mathematical Society, vol. 362, no. 6, pp. 3319-3363, 2010.

[6] A. Daniilidis, G. David, E. Durand-Cartagena, and A. Lemenant, "Rectifiability of self-contracted curves in the euclidean space and applications," Journal of Geometric Analysis, vol. 25, no. 2, pp. 1211-1239, 2015.

[7] A. Daniilidis, O. Ley, and S. Sabourau, "Asymptotic behaviour of self-contracted planar curves and gradient orbits of convex 
functions," Journal de Mathématiques Pures et Appliquées, vol. 94, no. 2, pp. 183-199, 2010.

[8] M. Longinetti, P. Manselli, and A. Venturi, "On steepest descent curves for quasi convex families in Rn," Mathematische Nachrichten, vol. 288, no. 4, pp. 420-442, 2015.

[9] M. Longinetti, P. Manselli, and A. Venturi, "On variational problems related to steepest descent curves and self-dual convex sets on the sphere," Applicable Analysis, vol. 94, no. 2, pp. 294307, 2015.

[10] T. Bonnesen and W. Fenchel, Theory of Convex Bodies, BCS Associates, 1987.

[11] R. Schneider, Convex Bodies: the Brunn-Minkowski Theory, vol. 44, Cambridge University Press, Cambridge, UK, 1993.

[12] B. de Finetti, "Sulle stratificazioni convesse," Annali di Matematica Pura ed Applicata, vol. 30, no. 4, pp. 173-183, 1949.

[13] H. W. Guggenheimer, Differential Geometry, McGraw-Hill, 1963.

[14] R. T. Rockafellar, Convex Analysis, vol. 28 of Princeton Mathematical Series, Princeton University Press, Princeton, NJ, USA, 1970. 


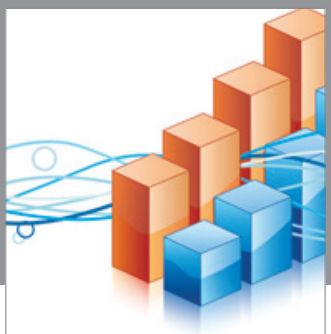

Advances in

Operations Research

vatem alat4

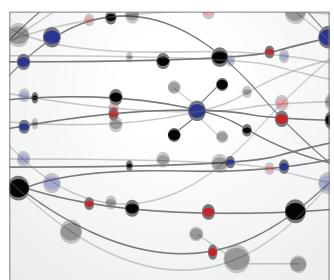

\section{The Scientific} World Journal
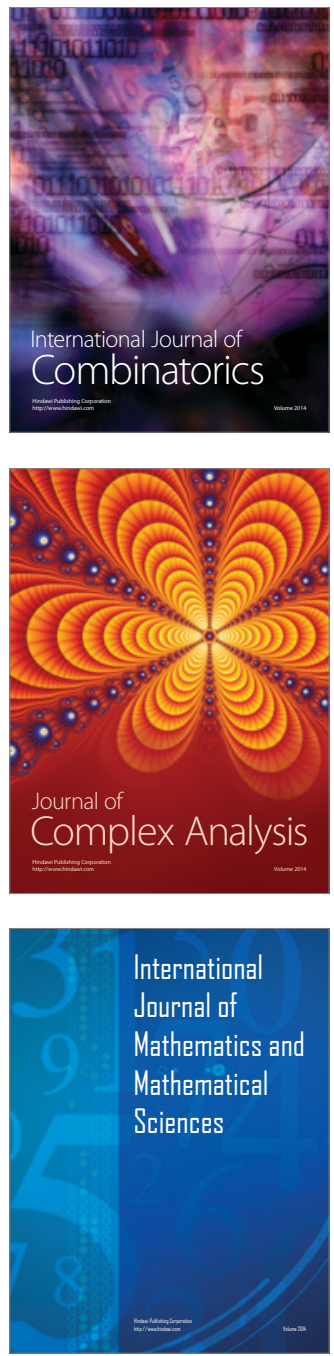
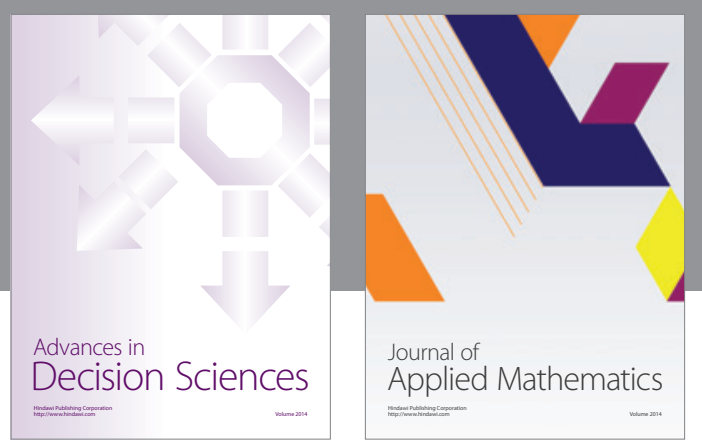

Algebra

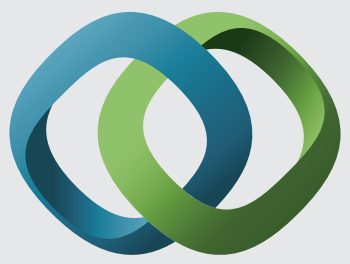

\section{Hindawi}

Submit your manuscripts at

http://www.hindawi.com
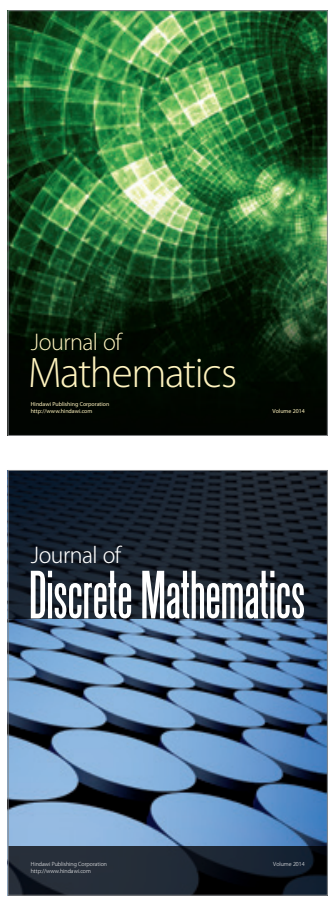

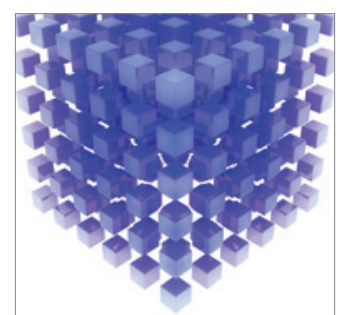

Mathematical Problems in Engineering
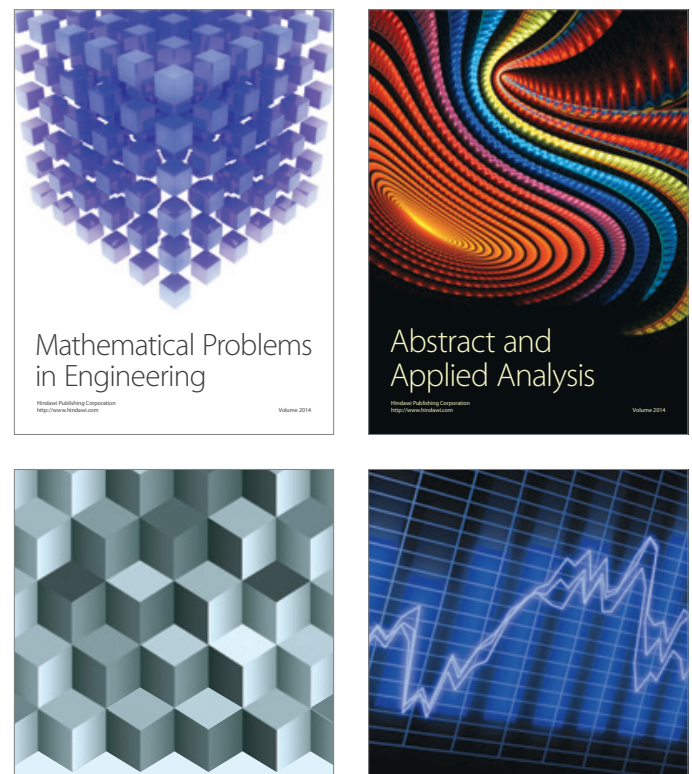

Journal of

Function Spaces

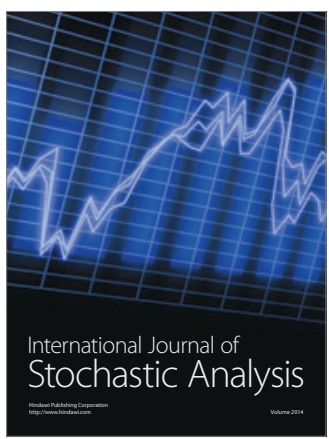

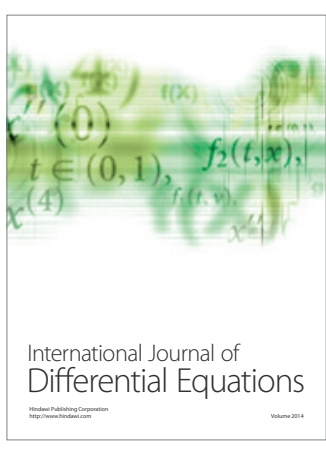
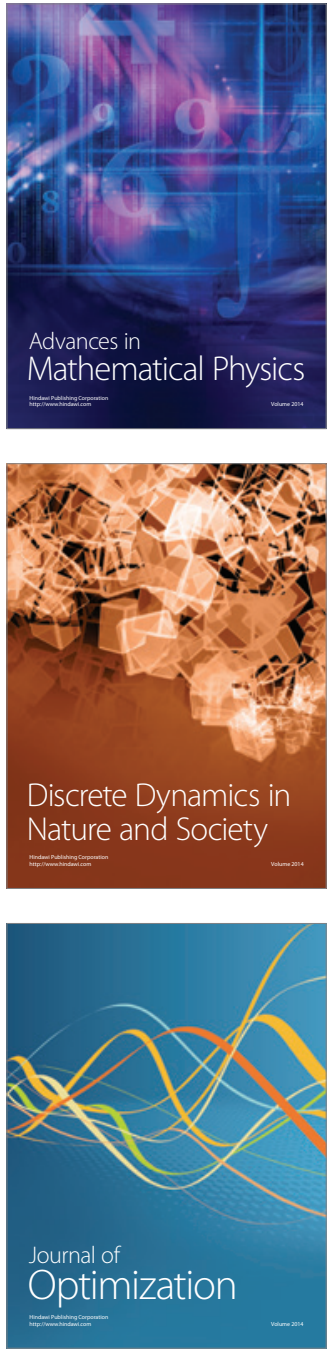